ANL/NDM-109

\title{
AN EVALUATED NEUTRONIC DATA FILE FOR BISMUTH*
}

\author{
by
}

P.T. Guenther, R.D. Lawson, J.W. Meadows, A.B. Smith,

D.L. Smith, and M. Sugimoto ${ }^{\dagger}$

ARGONNE NATIONAL LABORATORY

and

R.J. Howerton

LAWRENCE LIVERMORE NATIONAL LABORATORY

November 1989

Keywords

EVALUATION. Comprehensive evaluated neutronic data file for bismuth, $10^{-5} \mathrm{eV}$ to $20 \mathrm{MeV}$.

\author{
Engineering Physics Division \\ ARGONNE NATIONAL LABORATORY \\ 9700 South Cass Avenue \\ Argonne, IL 60439 \\ U.S.A.
}

\footnotetext{
* This work supported by the U.S. Department of Energy, under contract W-31-109-ENG-38.

$\dagger$ Permanent address: Japan Atomic Energy Research Institute, Tokai, Ibaraki, Japan.
} 


\section{NUCLEAR DATA AND MEASUREMENTS SERIES}

The Nuclear Data and Measurements Series presents results of studies in the field of microscopic nuclear data. The primary objective is the dissemination of information in the comprehensive form required for nuclear technology applications. This Series is devoted to: a) measured microscopic nuclear parameters, b) experimental techniques and facilities employed in measurements, c) the analysis, correlation and interpretation of nuclear data, and d) the evaluation of nuclear data. Contributions to this Series are reviewed to assure technical competence and, unless otherwise stated, the contents can be formally referenced. This Series does not supplant formal journal publication, but it does provide the more extensive information required for technological applications (e.g., tabulated numerical data) in a timely manner. 


\section{TABLE OF CONTENTS}

Page

List of Additional Titles

in the ANL/NDM Series

$\mathbf{v}$

Abstract vii

I. Introduction 1

II. Evaluated Total Cross Sections 1

III. Evaluated Elastic-Scattering

Cross Sections 4

Evaluated Inelastic-Scattering
Cross Sections

Evaluated Radiative-Capture
Cross Sections

VI. Evaluated $\left(n, 2 n^{\prime}\right)$ and $(n, 3 n)$ Reactions 19

VII. Evaluated Charged-Particle-Emitting Reactions 22

VIII. Evaluated Photon-Production Reactions 24

IX. Summary Comments 25

$\begin{array}{lr}\text { References } & 26\end{array}$

$\begin{array}{ll}\text { Appendix: Numerical-file Index } & 31\end{array}$ 


\title{
INFORMATION ABOUT OTHER ISSUES OF THE ANL/NDM SERIES
}

A list of titles and authors for all the previous issues appears in each report of the series. The list for reports ANL/NDM-1 through ANL/NDM-75 appears in ANL/NDM-76, and ANL/NDM-91 contains the list for reports ANL/NDM-76 through ANL/NDM-30. Below is the list for ANL/NDM-91 up to the current report. Requests for a complete list of titles or for copies of previous reports should be directed to:

\author{
Section Secretary \\ Applied Nuclear Physics Section \\ Engineering Physics Division \\ Building 316 \\ Argonne National Laboratory \\ 9700 South Cass Avenue \\ Argonne, Illinois 60439 \\ U.S.A.
}

ANL/NDM-91 A.B. Smith, P.T. Guenther and R.D. Lawson, On the Energy Dependence of the Optical Model of Neutron Scattering from Niobium, May 1985.

ANL/NDM-92 Donald L. Smith, Nuclear Data Uncertainties (Vol.-I): Basic Concepts of Probability, December 1988.

ANL/NDM-93 D.L. Smith, J.W. Meadows and M.M. Bretscher, Integral Cross-section Measurements for ${ }^{7} \mathrm{Li}\left(n, n^{3} t\right)^{4} \mathrm{He},{ }^{27} \mathrm{Al}(n, p){ }^{27} \mathrm{Mg},{ }^{27} \mathrm{Al}(n, \alpha){ }^{24} \mathrm{Na}$, ${ }^{58} \mathrm{Ni}(n, p){ }^{58} \mathrm{Co}$, and ${ }^{60} \mathrm{Ni}(n, p){ }^{60} \mathrm{Co}$ Relative to ${ }^{238} \mathrm{U}$ Neutron Fission in the Thick-target ${ }^{9} \mathrm{Be}(d, n){ }^{10} \mathrm{~B}$ Spectrum at $E_{\mathrm{d}}=7 \mathrm{MeV}$, October 1985.

ANL/NDM-94 A.B. Smith, D.L. Smith, P. Rousset, R.D. La wson and R.J. Howerton, Evaluated Neutronic Data File for Yttrium, January 1986.

ANL/NDM-95 Donald L. Smith and James W. Meadows, A Facility for High-intensity Neutron Irradiations Using Thick-target Sources at the Argonne Fast-neutron Generator, May 1986.

ANL/NDM-96 M. Sugimoto, A.B. Smith and P.T. Guenther, Ratio of the Prompt-fission-neutron Spectrum of Plutonium-239 to that of Uranium-235, September 1986.

ANL/NDM-97 J.W. Meadows, The Fission Cross Sections of ${ }^{230} T h,{ }^{232} T h,{ }^{233} U$,

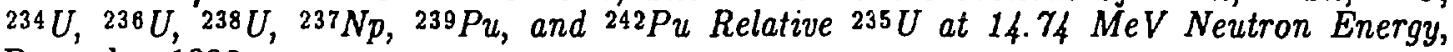
December 1986.

ANL/NDM-98 J.W. Meadows, The Fission Cross Section Ratios and Error Analysis for Ten Thorium, Uranium, Neptunium ane, Plutonium Isotopes at 14.74-MeV Neutron Energy, March 1987.

ANL/NDM-99 Donald L. Smith, Some Comments on the Effects of Long-range Correlations in Covariance Matrices for Nuclear Data, March 1987.

ANL/NDM-100 A.B. Smith, P.T. Guenther and R.D. Lawson, The Energy Dependence of the Optical-model Potential for Fast-neutron Scattering from Bismuth, May 1987. 
ANL/NDM-101 A.B. Smith, P.T. Guenther, J.F. Whalen and R.D. Lawson, Cobalt: Fast Neutrons and Physical Models, July 1987.

ANL/NDM-102 D. L Smith, Investigation of the Influence of the Neutron Spectrum in Determinations of Integral Neutron Cross-Section Ratios, November 1987.

ANL/NDM-103 A.B. Smith, P.T. Guenther and B. Micklich, Spectrum of Neutrons Emitted From a Thick Beryllium Target Bombarded With 7 MeV Deuterons, January 1988.

ANL/NDM-104 L.P. Geraldo and D.L. Smith, Some Thoughts on Positive Definiteness in the Consideration of Nuclear Data Covariance Matrices, January 1988.

ANL/NDM-105 A.B. Smith, D.L. Smith, P.T. Guenther, J.W. Meadows, R.D. Lawson, R.J. Howerton, and T. Djemil, Neutronic Evaluated Nuclear-Data File for Vanadium, May 1988.

ANL/NDM-106 A.B. Smith, P.T. Guenther, and R.D. Lawson, Fast-Neutron Elastic Scattering from Elemental Vanadium, March 1988.

ANL/NDM-107 P. Guenther, R. Lawson, J. Meadows, M. Sugimoto, A. Smith, D. Smith, and R. Howerton, An Evaluated Neutronic Data File for Elemental Cobalt, August 1988 .

ANL/NDM-108 M. Sugimoto, P.T. Guenther, J.E. Lynn, A.B. Smith, and J.F. Whalen, Some Comments on the Interaction of Fast-Neutrons with Beryllium, November 1988 .

ANL/NDM-109 P.T. Guenther, R.D. Lawson, J.W. Meadows, A.B. Smith, D.L. Smith, and M. Suc :moto, An Evaluated Neutronic Data File for Bismuth, August 1989 (this report).

ANL/NDM-110 D.L. Smith and L.P. Geraldo, A Vector Model for Error Propatation, March 1989.

ANL/NDM-111 J.E. Lynn, Fifty Years of Nuclear Fission, June 1989.

ANL/NDM-112 S. Chiba, P.T. Guenther, and A.B. Smith, Some Remarks on the Neutron Elastic- and Inelastic-Scattering Cross Sections of Palladium, May 1989. June 1989.

ANL/NDM-113 J.E. Lynn, Resonance Effects in Neutron Scattering Lengths, 


\title{
AN EVALUATED NEUTRONIC DATA FILE FOR BISMUTH
}

\author{
by \\ P.T. Guenther, R.D. Lawson, J.W. Meadows, A.B. Smith, \\ D.L. Smith and M. Sugimoto* \\ Argonne National Laboratory \\ and \\ R.J. Howerton \\ Lawrence Livermore National Laboratory
}

\begin{abstract}
A comprehensive evaluated neutronic data file for bismuth, extending from $10^{-5} \mathrm{eV}$ to $20.0 \mathrm{MeV}$, is described. The experimental database, the application of the theoretical models, and the evaluation rationale are outlined. Attention is given to uncertainty specification, and comparisons are made with the prior ENDF/B-V evaluation. The corresponding numerical file, in ENDF/B-VI format, has been transmitted to the National Nuclear Data Center, Brookhaven National Laboratory.
\end{abstract}

\footnotetext{
* Permanent address: Japan Atomic Energy Research Institute, Tokai, Ibaraki, Japan.
} 


\section{INTRODUCTION}

This report documents an elemental bismutb nuclear data file prepared for use in neutronic applications. Each of the following sections of this report: i) outlines the experimental database used in the evaluation (generally, the data search was closed in March 1988); ii) describes relevant calculational methods for interpolating and extrapolating experimental data, and for providing qualitative information where no experimental information is available; and iii) sets forth the evaluation methods and rationale used in creating the file. Attention is given to quantitative accuracies and uncertainty specifications where the experimental information is sufficiently comprehensive to warrant such considerations. The file is expressed in the ENDF/B-VI format ${ }^{1}$ and has been transmitted to the National Nuclear Data Center, Brookhaven National Laboratory. The present evaluation, and this document, address a file formulated in a manner consistent with the widest possible range of currently operational processing codes. A subsequent modification of the file will contain more complex formulations, particularly including energy-angle correlations of emitted neutrons, a feature presently not consistent with the majority of processing codes, and particle energy deposition necessary for Kerma determinations. Supplementary documentation will be provided for these addendums when the respective files are implemented. The primary objective is a practisal file for neutronic applications which reasonably summarizes the contemporary physical knowledge. There may be special applications for which this file will be deficient; for example, those involving activities and cross sections for isomeric states. Users interested in such special information should address special-purpose files.

Subsequent portions of this report deal with the following topics: Sec. II, total cross sections and resonance parameters; Sec. III, elastic-neutron scattering; Sec. IV, inelasticneutron scattering; Sec. V, radiative neutron capture; Sec. VI, $(n, 2 n)$ and $(n, 3 n)$ processes; Sec. VII, a variety of small charged-particle-emitting processes; Sec. VIII, photonproduction processes. Section IX summarizes the evaluation status and offers some guidance for future work. The appendix gives the explicit index of the unierical file.

\section{EVALUATED TOTAL CROSS SECTIONS}

\section{A. Resonance Region}

Resonance parameters are used to describe the neutron interaction to $100 \mathrm{keV}$. The parameters are taken from the work of Mughabghab, ${ }^{2}$, as modified by Mughabghab and Dunford for completeness, with small changes in the scattering radius to agree with experiment. A small background was introduced near $100 \mathrm{keV}$ to insure values matching the energy-averaged capture and total cross sections. The code RECENT ${ }^{3}$ was used to calculate the cross sections from the resonance parameters. For thermal energy, agreement with the values given in Ref. 2, within the stated uncertainty, was obtained for capture and elastic-scattering cross sections. Likewise, the calculation of the infinite-dilute resonance integral agreed with the value given in Ref. 2. The ENDF/B-V4 evaluation for bismuth has no resonance parameters for comparison. 


\section{B. Energy-Averaged and Unresolved-Resonance Cross Sections}

Above $0.1 \mathrm{MeV}$ the present evaluation uses a point-wise representation of the total cross section, with detailed resonance fluctuations to $2.0 \mathrm{MeV}$ and an energy-averaged behavior at higher energies. The evaluation is entirely based on experimental data assembled from the files of the National Nuclear Data Center and the literature. These sources are defined in Refs. 5 to 47 . The experimental data fluctuate at low energies. Therefore, the experimental results were averaged over energy increments of $10 \mathrm{keV}$ up to $0.2 \mathrm{MeV}$, of $50 \mathrm{keV}$ from 0.2 to $1.0 \mathrm{MeV}$, of $0.1 \mathrm{MeV}$ from 1.0 to $5.0 \mathrm{MeV}$, and of $0.2 \mathrm{MeV}$ above $5.0 \mathrm{MeV}$. Even in these averages some fluctuations persisted at the lower energies. The experimental uncertainties reported by the authors were propagated through the averaging procedures. The resulting averaged data sets were graphically compared with large plots. A few data sets were abandoned as being grossly inconsistent with the body of the information, or because vhey were outside the energy-range of interest. The very large majority of the uncertainties provided by the respective authors were only statistical. Thus, subjective judgments were used to provide systematic uncertainties. These judgments included considerations of the general reliability of the method, of the care used in the measurements, and of concurrent measurements of a reference standard (if any). The estimates were less reliable at lower energies where there are significant discrepancies, and where unknown self-shielding effects may be a factor. Reference citations 5 to 47 give an indication of energy range, systematic error, and acceptance for the evaluation.

The above energy-averaged database was evaluated using the statistical procedures of the code GMA.48 Thirty-five mesh points were used, subjectively selected between 0.3 and $20.0 \mathrm{MeV}$. These mesh points were chosen to give a reasonable description of the apparent gross features of the cross section. Fluctuations in the averaged cross sections made the GMA method unsuitable helow $0.3 \mathrm{MeV}$. The mesh-point values are given in Table 1. The GMA results will fluctuate slightly due to statistical artifacts. In addition, gross real fluctuations will persist within the context of the mesh selected at the input, for example, in the 3.0 to $4.5 \mathrm{MeV}$ region. The differentiation between the real effect and the statistical artifact is a matter of judjment.

In order to smooth the above statistical artifacts and to provide a vehicle for interpolation, the GMA results were fitted with a conventional spherical optical model over selected energy regions. Regions in which there were judged to be true gross fluctuations were avoided in the fitting procedure. The fitting generally varied the real and imaginary strengths, radii, and diffusenesses. The resulting potentials do not necessarily have any physical content; they merely provide a physically rational method for smoothing the GMA values. However, the potentials arrived at are not grossly different from those reported in fundamental physical studies. 49 In regions judged to show true gross structure, subjective estimates were used to smooth and interpolate the GMA results. Into the several-MeV region, detailed partially-resolved resonance structure has been reported, particularly in Refs. 8 and 32. Below $2.0 \mathrm{MeV}$, the best resolution data appear to be from Ref. 8 . Furthermore, averages of the data of Ref. 8 are in almost exact agreement with the GMA results. Therefore, the present evaluation uses the data of Ref. 8 to represent partially-resolved resonance structure with point-wise values to $2.0 \mathrm{MeV}$. It is evident from the data of Ref. 32 that partially-resolved resonance structure persists well above $2.0 \mathrm{MeV}$. However, the fluctuations are small (several percent), and they appear to have no applied importance; there is a motivation to keep the file as simple as practical, and there are limits on the content defined by ENDF format procedures. Thus, the present evaluation uses an energy-averaged representation above $2.0 \mathrm{MeV}$. 
Table 1. Energy-averaged Uncertainties. ${ }^{a}$

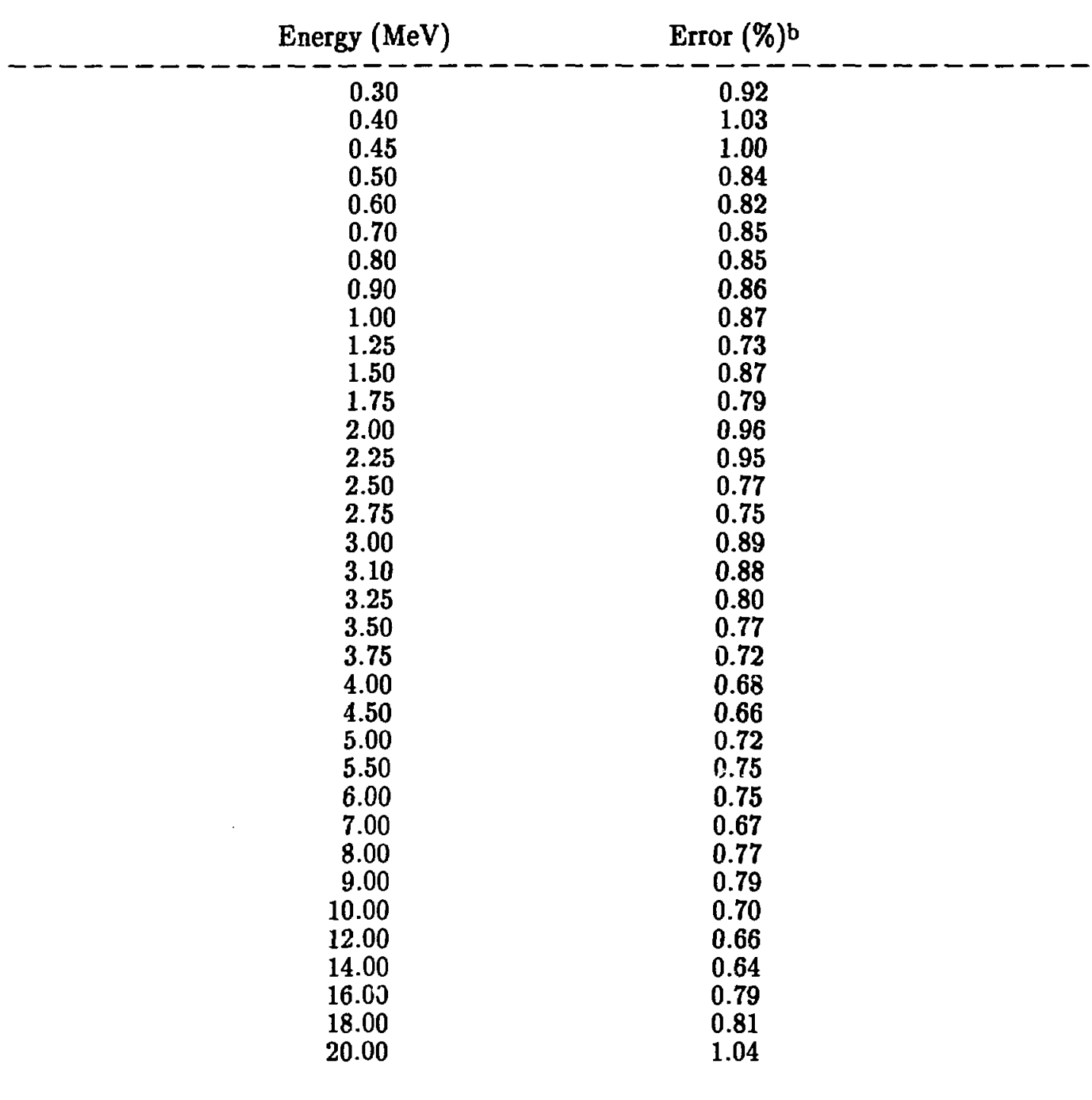

a GMA solution using 35 energy grid points. The correlation matrix is not shown.

b The numerical values given in the file are on a coarser grid as given in Table 2. 
The present energy-averaged evaluation is compared with the experimental data base in Fig. 1. The agreement is remarkably good. The structure in the 3.0 to $4.5 \mathrm{MeV}$ region is a notable feature. It is evident in a number of the data sets and, thus, is almost certainly not an experimental artifact. This structure has been a concern in fundamental physical interpretations, ${ }^{49}$ and is possibly attributable to quasi-particle processes. ${ }^{209} \mathrm{Bi}$ consists of one proton beyond the doubly closed shell at $\mathrm{A}=208$. ${ }^{207 \mathrm{~Pb}}$ is one neutron hole short of the same closed shell, and its total cross section shows gross structure somewhat similar to that of bismuth. The physical aspects of these observations will be discussed elsewhere. ${ }^{50}$ The energy-averaged uncertainties obtained from the 35 grid-point GMA solution are listed in Table 1 . They are only relevant to the energy average and do not extend below $0.3 \mathrm{MeV}$ due to fluctuations. They are selatively constant with energy and of small magnitude $(\approx 1 \%)$. These uncertainties may be somewhat optimistic, and they certainly depend upon the above-cited systematic uniertainty judgments. However, in regions of reasonably smooth cross section, where there are a number of extensive data sets, the agreement between the independent sets is of the order of $1 \%$ (e.g., between 2.0 and $6.0 \mathrm{MeV}$ ). The scatter of the database is larger below $\approx 1.5 \mathrm{MeV}$. This is partly due to fluctuations, but it is also likely that self-shielding perturbations are a contributing factor. It is noted that the better resolution measurements tend to lead to larger average cross-section values. Because of the rather small variation in the GMA-solution errors for the analysis with 35 grid points, it was decided to re-run the analysis with a much coarser grid structure involving only 12 energies. The results appear in Table 2, and these values are the basis for the uncertainty file $(\mathrm{MF}=33)$ provided with the numerical evaluated file. Fig. 2 illustrates the correlation pattern.

The present evaluation is qualitatively consistent with that of ENDF/B-V, as illustrated in Fig. 3. However, the present work has far more detail at lower energies, due to the introduction of new experimental information. The gross features at higher energies have not changed much. This is not surprising, as much of the experimental database is the same for both evaluations. The consistency of the data, and the resulting evaluation uncertainties, suggest a total cross section in the energy-averaged region that is really very well known to $\approx 15.0 \mathrm{MeV}$. Above $15.0 \mathrm{MeV}$ there are several isolated-energy experimental values, and one large data set. ${ }^{2}$ At lower energies the latter data set is in good agreemsit with the evaluated quantities, and there is no reason to believe that the quality of this set deteriorates with increasing energy above $15.0 \mathrm{MeV}$. However, it would be nice to have several more independent sets of experimental values above $15.0 \mathrm{MeV}$.

\section{EVALUATED ELASTIC-SCATTERING CROSS SECTIONS}

Below $0.1 \mathrm{MeV}$, the evaluated elastic-scattering cross sections follow from the above resonance parameters.

Above $0.1 \mathrm{MeV}$, the evaluation is explicitly based upon the work of Refs. 49 and 51 . Those two complementary references present an exhaustive fundámental study of elasticneutron scattering from bismuth, including detailed comparisons with the relevant observed quantities. The study results in two optical-statistical models, each of which gives a very good description of the observed elastic-scattering cross sections of bismuth to at least $20.0 \mathrm{MeV}$. The parameters of both models are energy dependent. One of the 


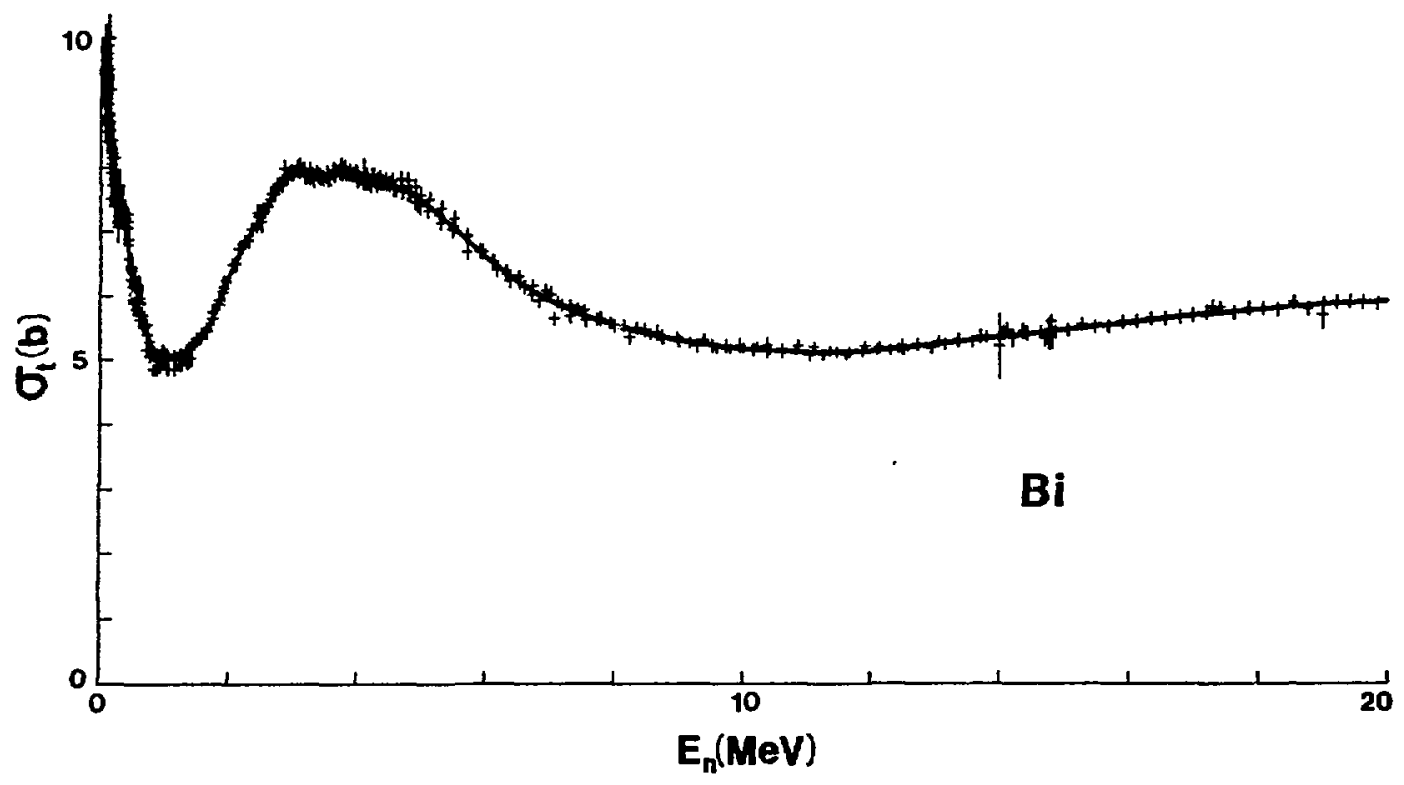

Fig. 1. Comparison of evaluated (curve) and measured (symbols) energy-averaged total cross sections of bismuth. 
Table 2. Coarse-Grid Covariance Matrix.a

Energy Grid and Asscsiated Errors.

$\begin{array}{ccc}\text { Group No. } & \text { Energy }(\mathrm{MeV}) & \text { Error(\%) } \\ 1 & 0.30 & 0.92 \\ 2 & 0.45 & 1.02 \\ 3 & 0.65 & 0.83 \\ 4 & 0.85 & 0.85 \\ 5 & 1.13 & 0.87 \\ 6 & 1.63 & 0.80 \\ 7 & 2.25 & 0.90 \\ 8 & 3.63 & 0.81 \\ 9 & 7.00 & 0.71 \\ 10 & 8.00 & 0.77 \\ 11 & 12.00 & 0.72 \\ 12 & 18.00 & 0.82\end{array}$

Correlation Matrix.
1.00
$\begin{array}{ll}0.33 & 1.00\end{array}$
$0.32 \quad 0.32$
1.00
$\begin{array}{lll}0.32 & 0.33 & 0.36\end{array}$
$\begin{array}{lll}0.32 & 0.33 & 0.37\end{array}$
1.00
$0.29 \quad 0.31$
$\begin{array}{ll}0.38 & 1.00\end{array}$
$\begin{array}{lll}0.35 & 0.35 & 1.00\end{array}$
$\begin{array}{lll}0.26 & 0.27 & 0.31\end{array}$
$\begin{array}{lll}0.32 & 0.34 & 0.34\end{array}$
$\begin{array}{lll}0.26 & 0.29 & 0.33\end{array}$
0.34
0.36
$\begin{array}{lll}0.36 & 0.37 & 1.00\end{array}$
$\begin{array}{lll}0.28 & 0.30 & 0.34\end{array}$
$\begin{array}{lllll}0.36 & 0.37 & 0.38 & 0.39 & 0.43\end{array}$
$\begin{array}{lllll}0.37 & 0.38 & 0.38 & 0.39 & 0.45\end{array}$
1.00
$\begin{array}{lll}0.28 & 0.31 & 0.35\end{array}$
$0.37 \quad 0.39$
$\begin{array}{lll}0.39 & 0.39 & 0.44\end{array}$
$0.46 \quad 1.00$
$\begin{array}{lll}0.28 & 0.31 & 0.35\end{array}$
$\begin{array}{lllll}0.34 & 0.35 & 0.35 & 0.35 & 0.38\end{array}$
$\begin{array}{lll}0.47 & 0.46 & 1.00\end{array}$
$\begin{array}{llll}0.41 & 0.42 & 0.40 & 1.00\end{array}$

- - - - - - - - - - - - - - - - - - - - - - - - - - - - - - - - -

a GMA solution using 12 grid points. The uncertainty file $(M F=33)$ of this evaluation is based upon these results. 


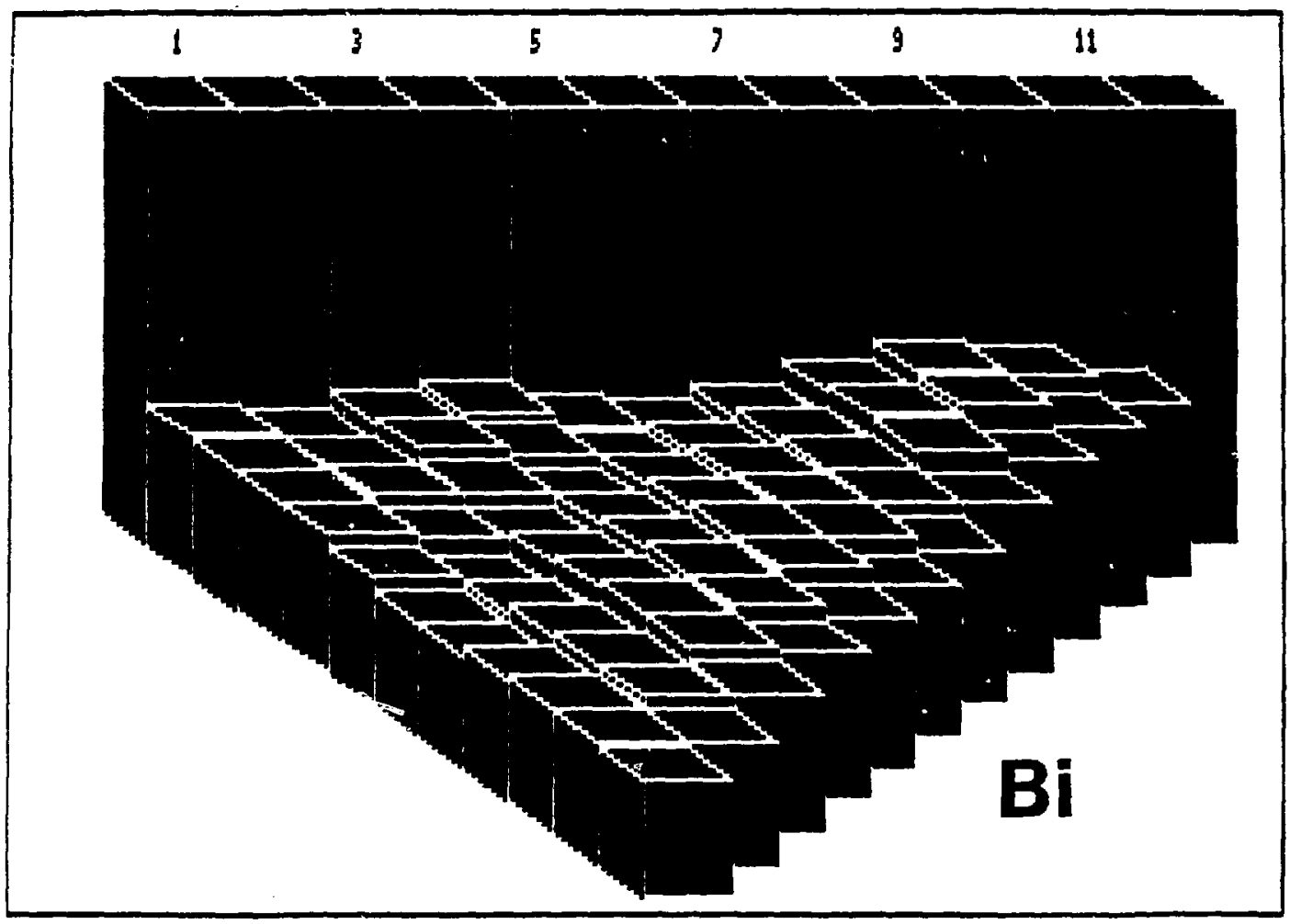

Fig. 2. Graphical presentation of the correlation matrix from Table 2. 


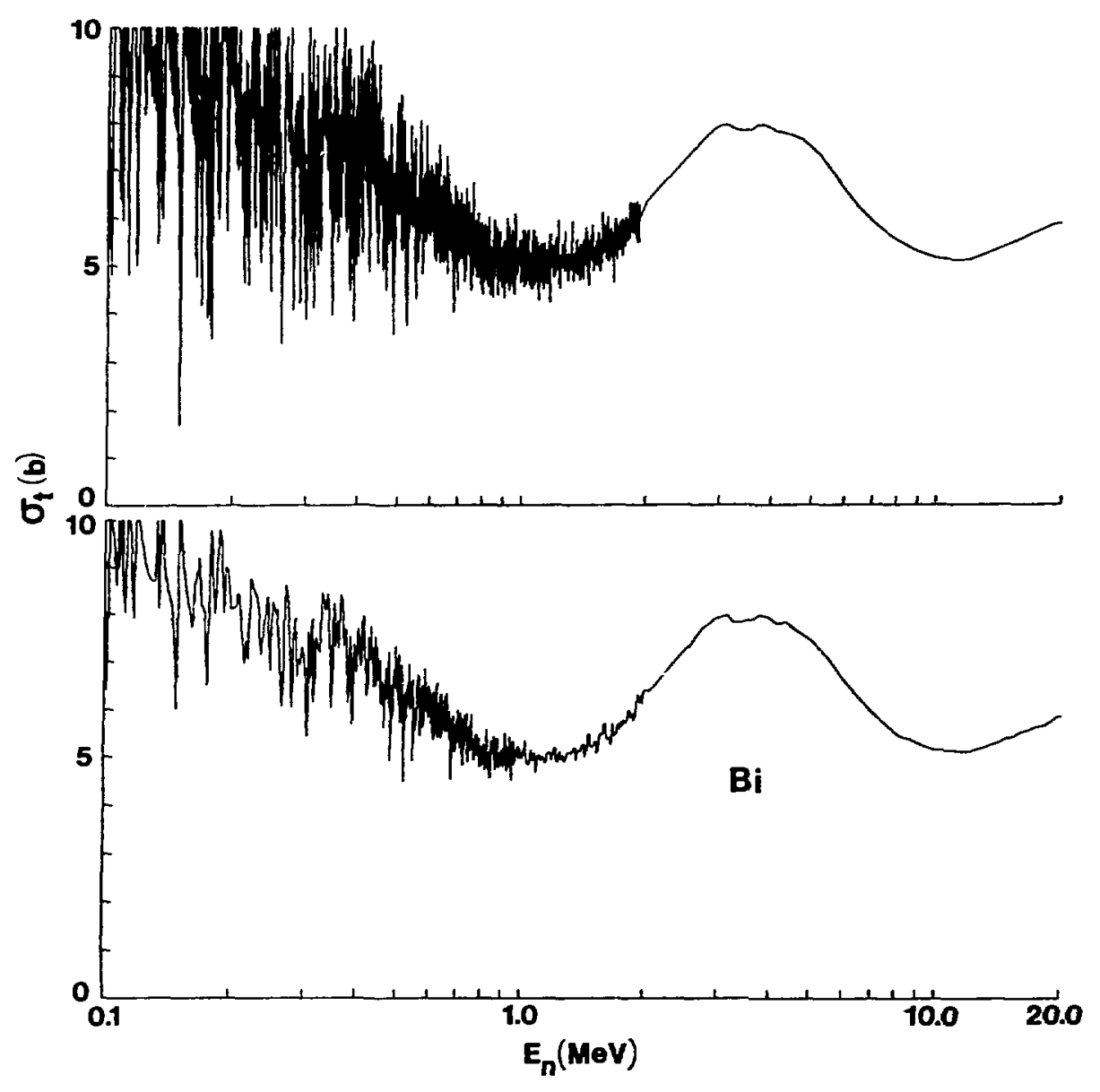

Fig. 3. Comparison of the present evaluated total cross sections (upper portion of the figure) with those of ENDF/B-V (lower portion of the figure). For clarity, the cross sections are truncated to a maximum of $10 \mathrm{~b}$. 
models is based upon a "conventional" potential consisting of a Saxon-Woods real term and a Saxon-Woods-derivative imaginary term. The other model adds a surface component to the real Saxon-Woods potential, as required by the dispersion relationship. ${ }^{52}$ The former potential was used in the present evaluation. It is not as physically sound as the potential that includes the real surface component, but it gives essentially an equivalent parameterization of the elastic scattering, and is easier to implement in practice.

The calculations used the excitation energies and $\mathrm{J}^{\pi}$ values of Table 3.53 An energy mesh suitable for defining the gross energy dependence of the cross section was used. The

Table 3. Excitation energies and $\mathrm{J}^{\pi}$ values used

in determining the evaluated elastic-scattering cross sections. ${ }^{53}$

\begin{tabular}{cc}
\hline $\mathrm{E}_{\mathrm{x}}(\mathrm{MeV})$ & $\mathrm{J}^{\pi}$ \\
\hline $0.000(\mathrm{~g} . \mathrm{s})$. & $9 / 2^{-}$ \\
0.897 & $7 / 2^{-}$ \\
1.609 & $13 / 2^{+}$ \\
2.430 & $1 / 2^{+}$ \\
2.492 & $3 / 2^{-}$ \\
2.563 & $9 / 2^{+}$ \\
2.582 & $7 / 2^{+}$ \\
2.599 & $11 / 2^{+}$ \\
2.601 & $13 / 2^{+}$ \\
2.616 & $5 / 2^{+}$ \\
2.741 & $15 / 2^{+}$ \\
2.766 & $3 / 2^{+}$ \\
2.822 & $5 / 2^{-}$ \\
2.847 & $1 / 2^{+}$ \\
2.919 & $1 / 2^{+}(?)$ \\
2.957 & $3 / 2^{+}(?)$ \\
2.986 & $19 / 2^{+}$ \\
3.038 & $5 / 2^{+}$ \\
3.091 & $7 / 2^{+}$ \\
3.118 & $1.5^{-}$
\end{tabular}

calculations also provided the neutron total cross sections. The latter agreed with those of the evaluation to within $<\approx 3 \%$ throughout the energy range. The calculated elasticscattering results were renormalized by the respective small percentages to bring the calculated and evaluated total cross sections into exact agreement. This procedure propagates the gross structure of the evaluated total cross section into the elastic cross section (e.g., in the 3.0-5.0 MeV range). In addition, it was assured that the evaluated results were consistent with "Wick's Limit". 54

The energy-averaged evaluated elastic-scattering results are illustrated in Fig. 4. They, together with the total cross section, imply the illustrated nonelastic cross section. Interestingly, the latter peaks at $10.0-12.0 \mathrm{MeV}$ and then decreases to somewhat smaller values at higher energies. This behavior is, in part, a reflection of the changes in the 


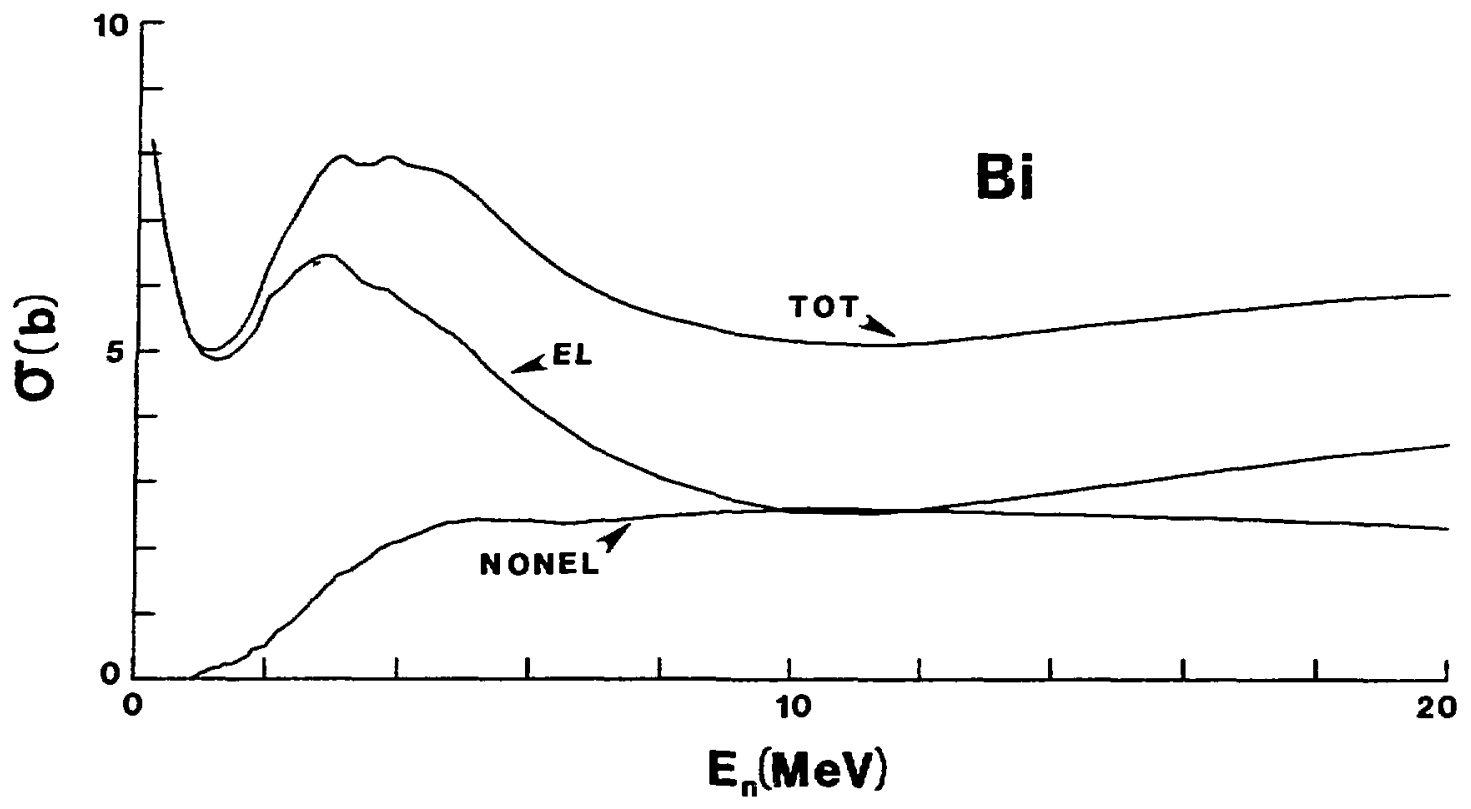

Fig. 4. The present energy-averaged evaluated total, elastic-scattering and nonelastic cross sections. 
underlying physical model for energies $16.0-18.0 \mathrm{MeV}$ above the Fermi energy, as discussed in Refs. 49 and 51 . Those changes will significantly affect the continuum inelasticscattering cross section, as the latter is determined by the difference between the total cross section and the other partial cross sections. The corresponding evaluated differential elastic-scattering cross sections of bismuth are illustrated in Fig. 5. In the numerical file, they are expressed in the form of Legendre-polynomial expansions

$$
\left(\mathrm{P}_{\ell} ; \ell=0, \mathrm{n}\right) \text {, with } \mathrm{n} \leq 20 .
$$

The final evaluated elastic-scattering cross section was constructed so as to retain all of the resonance fluctuations of the total cross section, while at the same time following the energy-averaged values outlined above. This is a reasonable approximation, as the elastic-scattering cross section is by far the largest component of the total cross section in the region where resonance fluctuations are prominent. Given the available experimental information, and the requirement of exact internal file consistency, there is little alternative to pursuing this approximate representation of resonance fluctuations in the elastic-scattering cross section.

The above elastic-scattering evaluation is a complex mixture of observations, models, and physical theory. As such, it is very difficult to quantify uncertainties. A reasonable guideline is $3-5 \%$ uncertainty on the angle-integrated elastic-scattering cross section above $\approx 1.6 \mathrm{MeV}$, and the uncertainty of the total cross section at lower energies. Correlations will be strong, but they cannot be reasonably estimated.

The present elastic-scattering evaluation is in very good agreement with that of ENDF/B-V,4 as illustrated in Fig. 6. The agreement is remarkable, in view of the fact that the bismuth ENDF/B-V evaluation was very rapidly assembled to meet a fusionhybrid data need at a time when there existed no prior ENDF file for bismuth. Additional measurements above $\approx 10.0 \mathrm{MeV}$ have the potential for improving the present evaluation, as discussed in Refs. 49 and 51. In particular, the change in the potential for energies 16.018.0 MeV above the Fermi energy should be better defined.

\section{EVALUATED INELASTIC-SCATTERING CROSS SECTIONS}

\section{A. Discrete Inelastic-Scattering Processes.}

The experimental database was assembled from the files of the National Nuclear Data Center. It is relatively limited, with only the citations of Refs. 55-64. An additional search of the literature turned up several more experimental results, but numerical values were not available in these cases and, thus, that experimental information was not used. Only five of the experimental data sets resulted from direct $\left(n, n^{\prime}\right)$ measurements, the remainder of the inelastic-scattering cross sections being construed from the results of $\left(n ; n^{\prime}, \gamma\right)$ measurements. The latter were reasonably consistent near the respective thresholds, but varied a great deal at energies well above threshold. This is probably the consequence of differing interpretations of the various branching ratios. The $\left(n, n^{\prime}\right)$ results extended to excitations of only $\approx 3.0 \mathrm{MeV}$, and the inelastically-scattered neutrons were uniquely defined only for the excitation of the first two levels. The observed higher-energy excitations were composites of contributions from several levels. The $\left(n, n^{\prime}\right)$ results of 


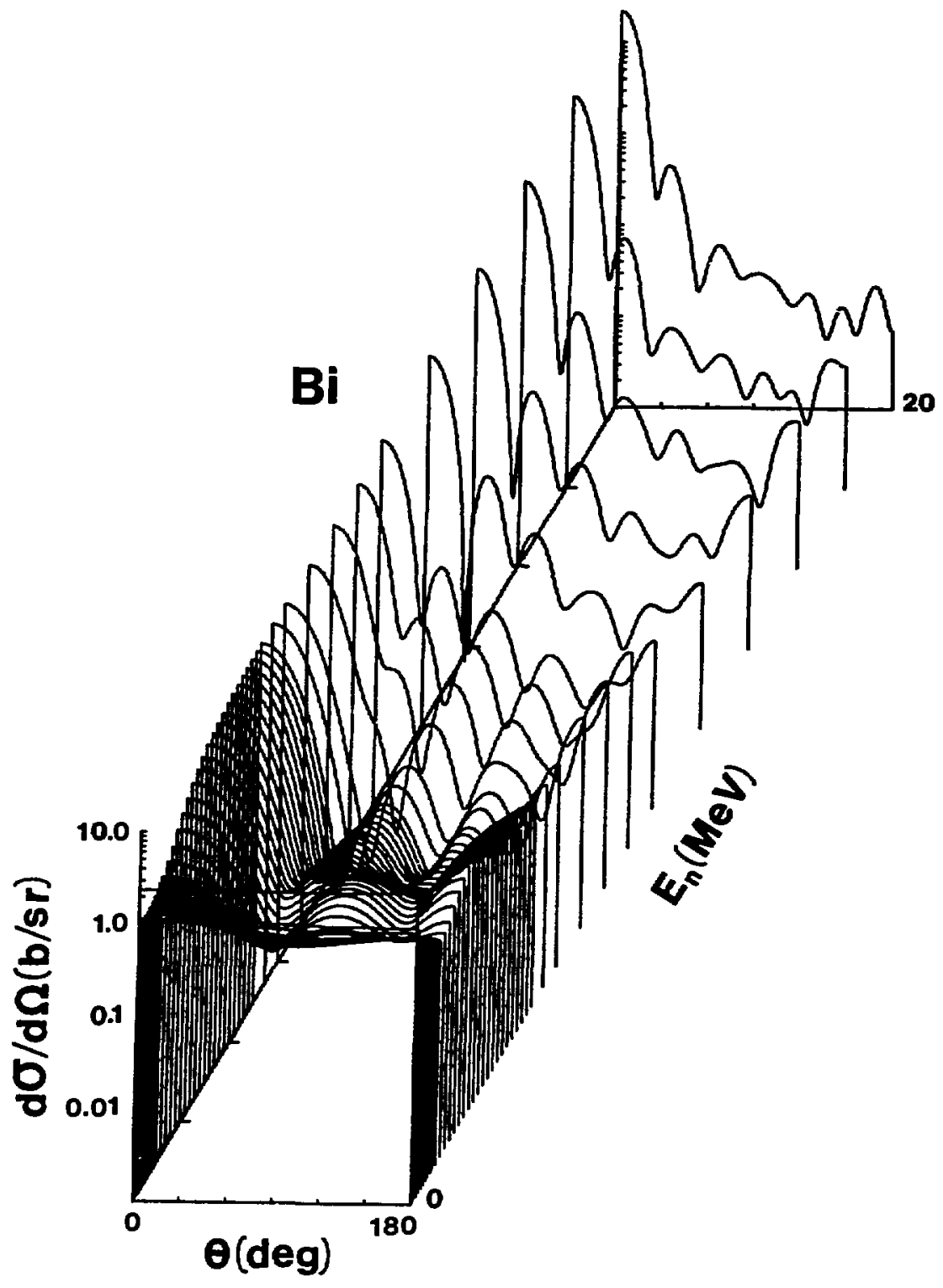

Fig. 5. The present evaluated differential elastic-scattering cross sections (expressed in the laboratory coordinate system). 


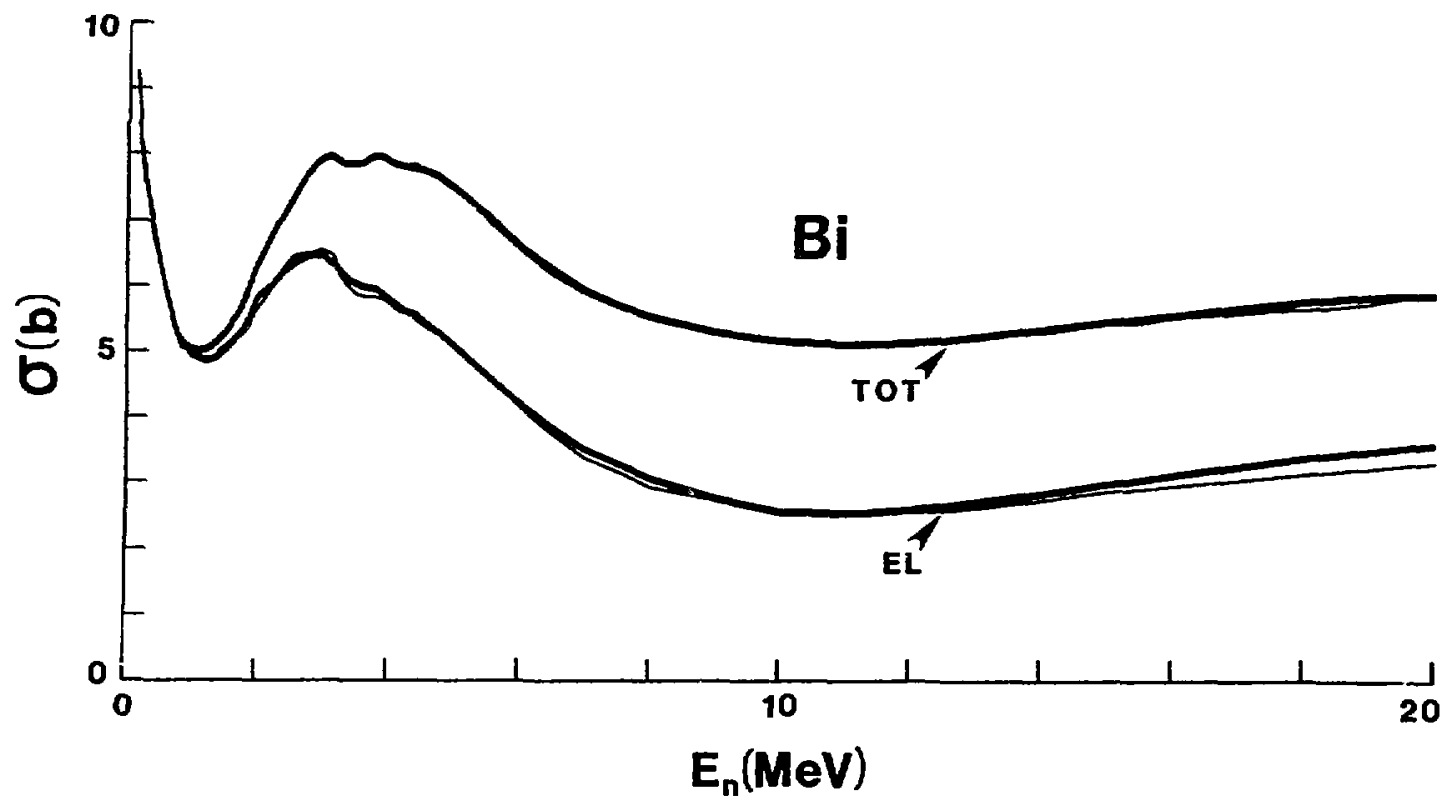

Fig. 6. Comparison of the present evaluated energy-averaged total and elasticscattering cross sections (heavy curves) with those of ENDF/B-V4 (light curves). 
Ref. 59 were very much larger than those of other similar types of measurements and, generally, much larger than the results of $\left(n ; n^{\prime}, \gamma\right)$ measurements. Thus, they were abandoned. The $\left(n, n^{\prime}\right)$ results of Ref. 61 were, in contrast, much smaller than the body of the experimental results. Therefore, they were also abandoned. The remaining database consists of the values from only three $\left(n, n^{\prime}\right)$ studies ${ }^{55,63104}$, and the rather scattered results of five $\left(n ; n^{\prime}, \gamma\right)$ measurements, some of which are very old. This is not an auspicious experimental foundation for an evaluation.

In view of the above unfortunate experimental situation, the evaluation used the potential of Ref. 49, the nineteen excited levels of Table 3, and the optical-statistical model to calculate the discrete inelastic-excitation cross sections. At excitation energies of greater than $3.118 \mathrm{MeV}$, the calculations assumed a continuum of levels given by a modified Gilbert and Cameron ${ }^{65}$ statistical representation, as defined in Ref. 49 . The resulting calculated excitation cross sections were appropriately combined to make possible a direct comparison with the experimental information. The results of $\left(n, n^{\prime}\right)$ measurements ${ }^{63}, 64$ and the calculated cross sections for the excitation of the first $t$ wo levels compared reasonably well. There was similar agreement between calculated and observed excitations of the composite of levels at $\approx 2.55 \mathrm{MeV}$ up to incident-neutron energies of $\approx$ 4:0 MeV. At higher energies, the results obtained in the recent measurements of Ref. 55 were significantly larger than those calculated with the statistical model. Thus, above 4.0 MeV, the evaluated cross sections for the excitation of levels at $\approx 2.55 \mathrm{MeV}$ were taken from the measured values of Ref. 55. The distribution of the measured cross section between the various levels making up the composite observed value near $\mathrm{E}_{\mathrm{x}}=2.55 \mathrm{MeV}$ is not known, but is generally of no applied importance. There was also reasonable agreement between calculatea and measured $\left(n ; n^{\prime}, \gamma\right)$ cross sections near thresholds. Thus, the evaluated file used the calculated results, adjusted in the region of $2.55 \mathrm{MeV}$ excitations as described above, leading to the cumulative inelastic-scattering cross sections shown in Fig. 7. It was assumed that these are in general, statistical processes where the neutron emission is symmetric about $90^{\circ}$ and is very nearly isotropic. Therefore, the evaluation assumes isotropic neutron emission as a result of the excitation of discrete levels.

The present evaluation has far more detail than that of ENDF/B-V, containing thirteen more excited states. However, the cumulative magnitudes of the discrete excitations are similar in the two files. In the combined total, the present evaluation of discrete inelastic-scattering cross sections is estimated to be reliable to better than $10 \%$ for the energy averages in regions of appreciable cross section. The uncertainties are larger where the cross sections are smaller and near threshold. Of course, the present evaluation does not display the structure at lower energies reported in some measurements.03 Such structure was addressed in ENDF/B-V, but it is of small magnitude. Moreover, the experimental evidence is not sufficient to provide very good definition, nor is the resolution equivalent to that of the much larger structure observed in the total cross section. Therefore, these fluctuations are given no attention in the present evaluation.

The contemporary experimental database is certainly marginal. Extensive new measurements will be required if the evaluation is to be significantly improved. These will not be simple, as the density of levels above excitations of $\approx 2.5 \mathrm{MeV}$ is considerable. 


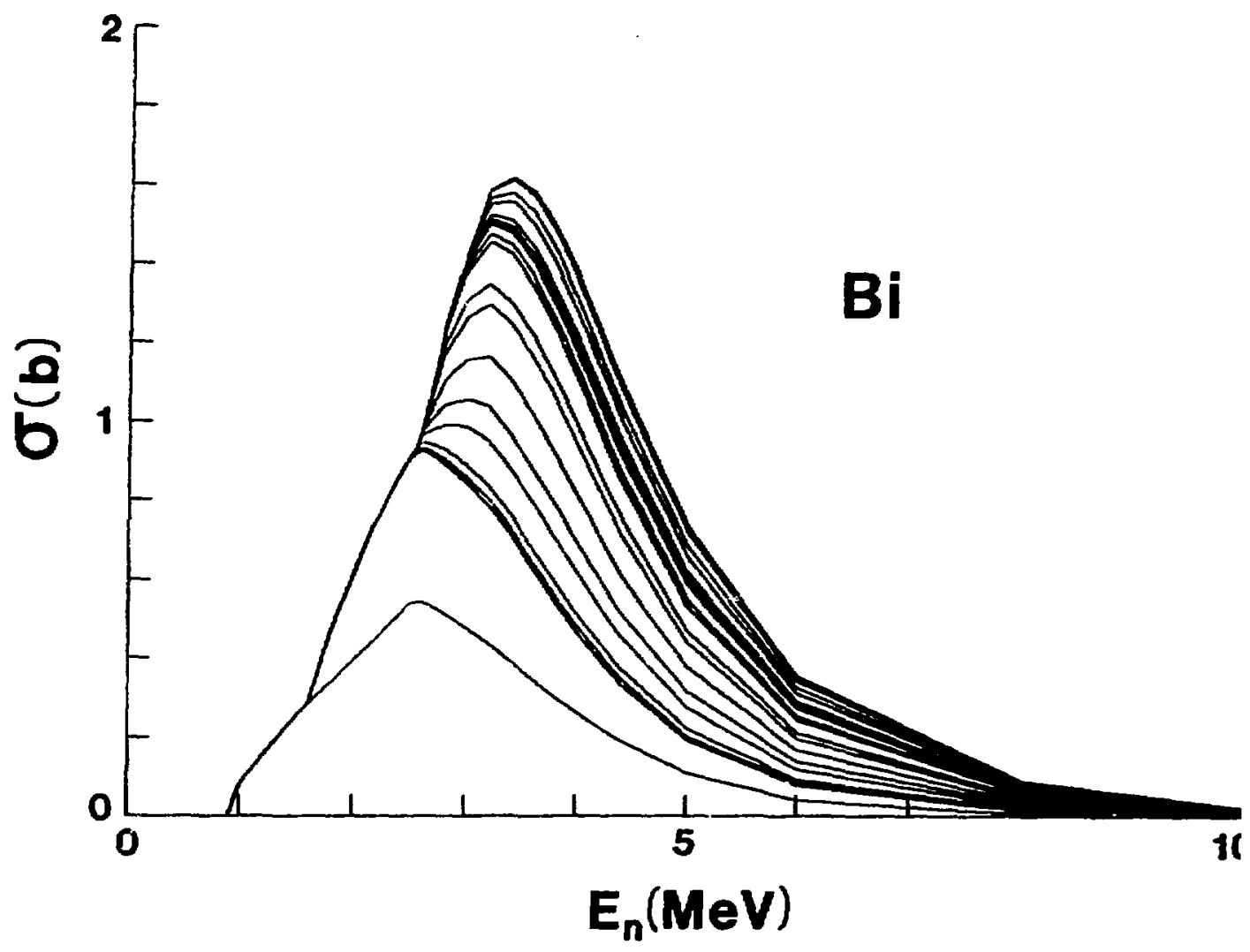

Fig. 7. Cumulative sum of the evaluated discrete inelastic-scattering cross sections of elemental bismuth. 


\section{B. Continuum Inelastic-Scattering Processes.}

The magnitude of the continuum inelastic-scattering cross section was defined by the difference between the neutron total cross section and the other partial cross sections. This assures the internal consistency of the file. This cross section rises from the threshold to the $(n, 2 n)$ threshold, and then it rapidly falls as the $(n, 2 n)$ and $(n, 3 n)$ cross sections increase. Finally, at higher energies, it approaches an approximately constant value. This general behavior is indicated in Fig. 8. The uncertainties reflect those of the total, elastic-scattering, $(n, 2 n)$ and $(n, 3 n)$ cross sections. The present continuum inelastic-scattering cross section is quite similar to that given in ENDF/B-V.4

The neutron spectra emitted as a result of continuum inelastic scattering were calculated using the methods described in Ref. 66, and were verified against the direct measurements of Ref. 67. The calculations employed the computer codes CADE68 and ALICE ${ }^{89}$ in a manner similar to that described in Ref. 66 . The details of the procedure are given in that reference. In the present evaluation form, the file assumes isotropy of neutron emission. The assumption is a simplification that makes the file usable with most contemporary processing codes, and it will not significantly affect the physical results in the majority of applications as, to at least $10 \mathrm{MeV}$, the experimentally observed spectra are essentially isotropic with little precompound contribution. ${ }^{67}$ A future formulation of the file will contain angle-energy correlations at higher energies, and this will be described elsewhere. ${ }^{70}$ The calculations also provide the continuum neutron-emission spectra relevant to other reactions of the $\left(n ; n^{\prime}, X\right)$ typt, as discussed below.

In view of the lack of detailed experimental information and the extensive use of model extrapolations in the above evaluation of inelastic scattering, uncertainty estimates can be no more than qualitative, as noted above. The corresponding numerical uncertainties given in the formal file should only be taken in that qualitative spirit.

\section{EVALUATED RADIATIVE-CAPTURE CROSS SECTIONS}

The experimental data for fast-neutron radiative capture in bismuth are sparse and conflicting, as shown in Fig. 9.71-83 Moreover, fluctuations lead to large variations in the experimental results below several-hundred keV. Below $100 \mathrm{keV}$, the evaluation is based upon the resonance parameters described above. Above $100 \mathrm{keV}$, a relatively smooth energy-averaged behavior was assumed. With the uncertain database, the energyaveraged evaluation relies upon a calculated result. The calculations were based upon a simple dipole model, 84 normalized to the $S_{0}$ strength function given in Ref. 2. The potential used in the calculation was that of Ref. 49 , and the calculations considered competition from the inelastic excitation of 19 levels and a continuum of excited levels, as given in Ref. 53 and Table 3. The calculated result, shown in Fig. 9, is in reasonable agreement with the measured values from $\approx 0.5-1.0 \mathrm{MeV}$, where the experimental results are fairly consistent. Furthermore, the calculated results properly account for competition from inelastic-scattering channels, and, by definition, are consistent with the $\boldsymbol{S}_{0}$ strength function. A small direct-capture resonance was included near $14.0 \mathrm{MeV}$, though the magnitudes of the cross sections in that region are so small that the effect is largely of a cosmetic nature. 


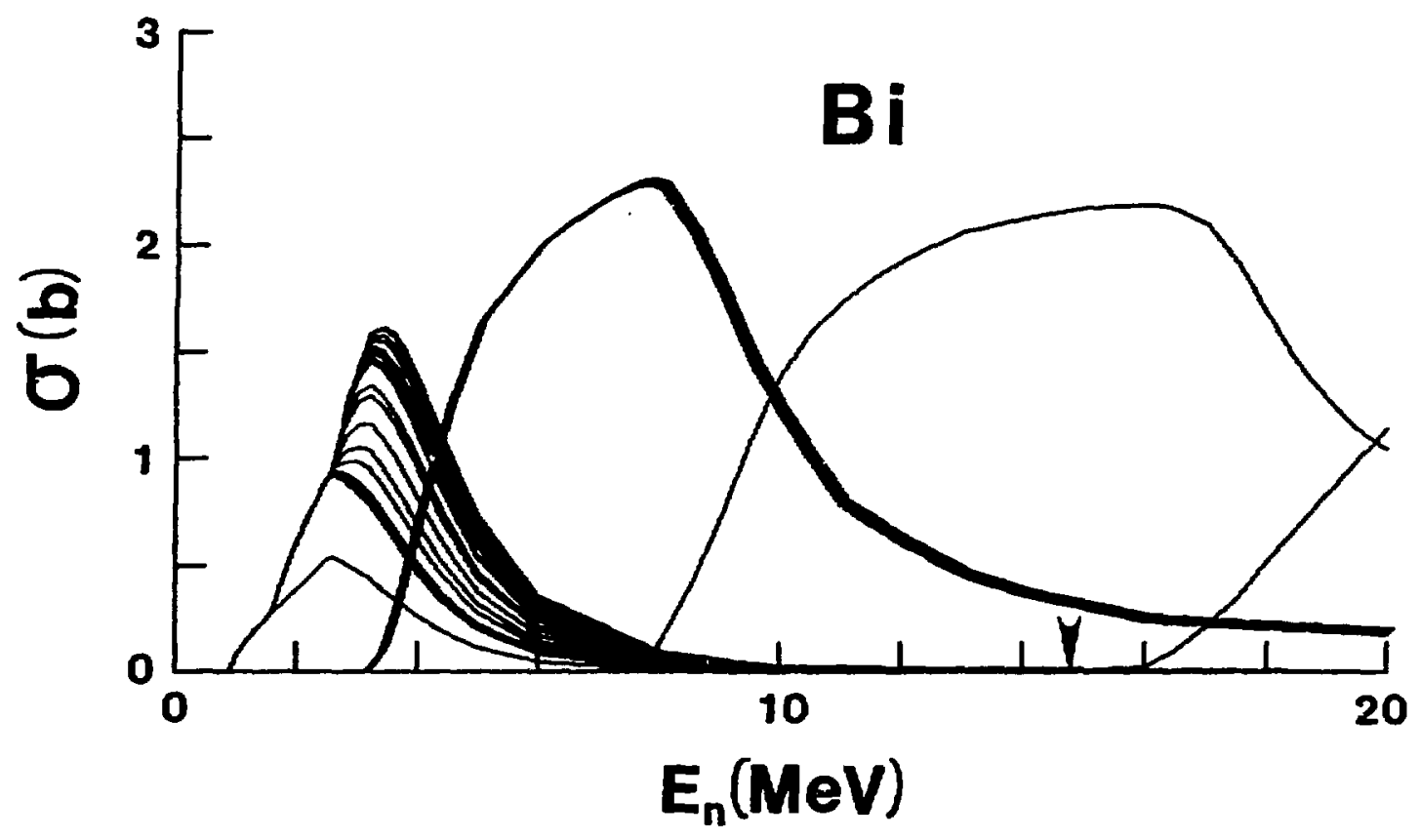

Fig. 8. The evaluated continuum inelastic-scattering cross section (heavy curve) compared with the cumulative sum of the discrete-inelastic components and with the $(n, 2 n)$ and $(n, 3 n)$ cross sections (light curves). 


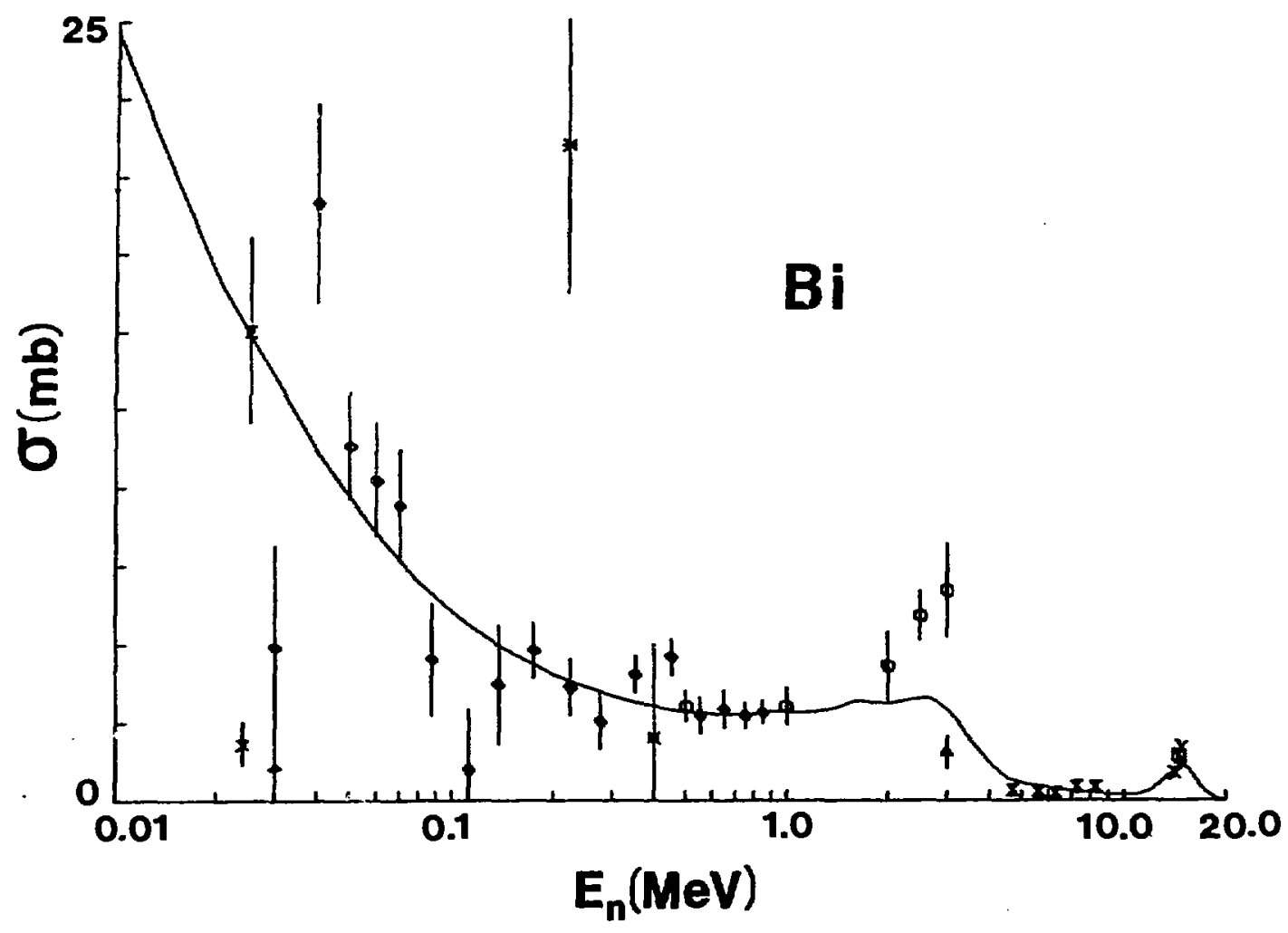

Fig. 9. Comparison of measured (symbols) and evaluated (curve) neutron radiative capture cross sections of bismuth. 
The present evaluation is generally larger than that of ENDF/B-V by $25-50 \%$. However, the cross sections are very small above $0.1 \mathrm{MeV}(<\approx \mathrm{a}$ few $\mathrm{mb}$ ). The uncertainties associated with the present evaluation are large, perhaps $25 \%$ or more. These large quaiitative uncertainties are given in the numerical file, but are probably not of an applied concern in view of the very small magnitude of the cross section. The evaluated results cannot be significantly improved until better and more extensive experimental information becomes available.

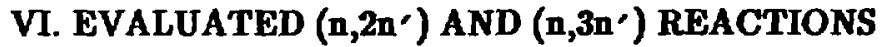

The experimental microscopic database available for the $\left(n, 2 n^{\prime}\right)$ evaluation is limited to the information of Refs. 85-93. These data consist of individual measurements in the vicinity of $14 \mathrm{MeV}$, and the two far more comprehensive data sets of Refs. 85 and 89, both of which were obtained using prompt-detection techniques. Reference 89 gives very good coverage from threshold to $\approx 14 \mathrm{MeV}$, while Ref. 85 gives similar good coverage from $\approx 14 \mathrm{MeV}$ to $20 \mathrm{MeV}$. These two major sets are relatively consistent in the common $14 \mathrm{MeV}$ region. The available experimental database is summarized in Fig. 10.

The above database is not sufficient for statistical evaluation procedures; thus, subjective judgment was used in constructing this evaluation, guided by statistical calculations using the code CADE. 98 The calculated $\left(n, 2 n^{\prime}\right)$ cross section had an energy-dependent shape very similar to that indicated by the measured values of Reis. 85 and 89 , but a magnitude $\approx 8 \%$ larger than the measured values at the maximum of the cross section in the 14-16 MeV region. As shown in Fig. 10, the evaluation smoothly follows the measured values of Ref. 89 ( + ) from threshold to $14 \mathrm{MeV}$, and those of Ref.85 (a) from $14-20 \mathrm{MeV}$. The result is consistent with the more precise of the isolated $\approx 14-\mathrm{MeV}$ experimental results. Subjective estimates of the evaluation uncertainties are given in Table 4. They reflect the experimental uncertainties of Fefs. 85 and 89 . The corresponding neutron-emission spectra were determined from the combined model interpretations outlined above in the context of continuum inelastic-neutron emission.

Table 4. $\left(n, 2 n^{\prime}\right)$ Cross Section Uncertainties.

$\mathrm{E}_{\mathrm{n}}(\mathrm{MeV}) \quad$ Uncertainty(\%)

$\begin{array}{rr}9.0 & 15 \\ 10.0 & 6 \\ 12.0 & 5 \\ 14.0 & 5 \\ 16.0 & 5 \\ 18.0 & 5 \\ 20.0 & 6\end{array}$




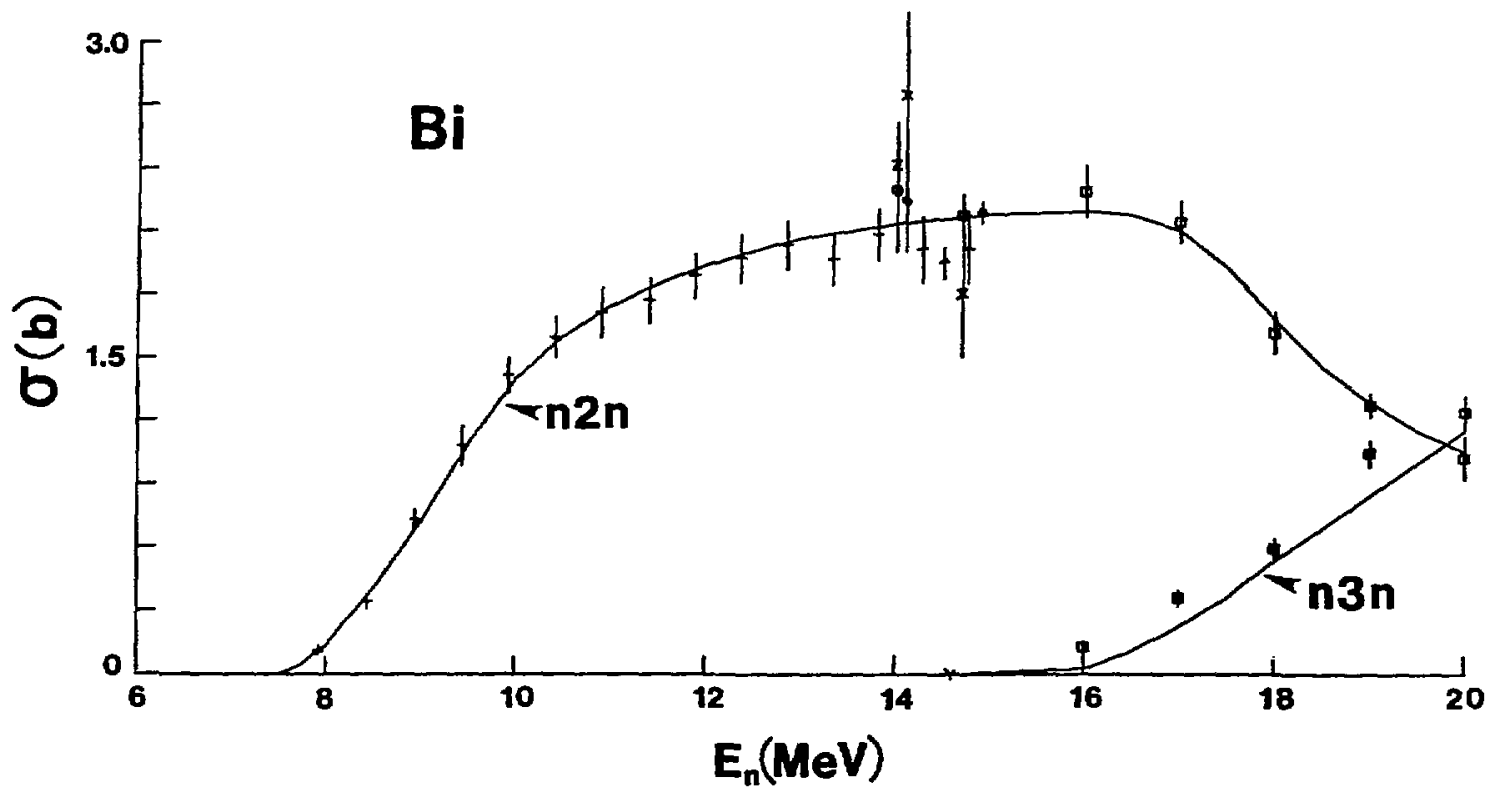

Fig. 10. Measured and evaluated $\left(n, 2 n^{\prime}\right)$ and ( $\left.n, 3 n^{\prime}\right)$ cross sections. Symbols indicate experimental values, and the curves the results of the present evaluation. 
The present evaluated $\left(n, 2 n^{\prime}\right)$ cross sections are very similar to those given in ENDF/B-V.4 The two evaluations are frequently consistent within the uncertainties attributed to the present evaluation alone. Though the evaluations were done entirely independently, the agreement is not surprising as the database has not changed in the intervening years, and both evaluations are based upon the experimental data. In both cases, the $\left(n, 2 n^{\prime}\right)$ cross section is very large-well over two barns at the maximum-and thus bismuth should be a good neutron multiplier in fusion applications.

There are only two data sets relevant to the $\left(n, 3 n^{\prime}\right)$ cross section, a single value near threshold from Ref. 94 and the five values from Ref. 85, distributed between 16-20 MeV. CADE-calculated results are reasonably descriptive of the measured values, but are somewhat lower in the $16-18 \mathrm{MeV}$ region. The present evaluation is a compromise between the experimental values of Ref. 85 and the CADE-calculated results. This compromise was chosen because the simple sum of the $\left(n, 2 n^{\prime}\right)$ and $\left(n, 3 n^{\prime}\right)$ results of Ref. 85 produces a physically unrealistic "bump" in the nonelastic cross section in the 16-18 MeV region, or a similarly unphysical dip in the continuum inelastic-scattering cross section. The effect is largely cosmetic in the context of neutronic applications, as it represents $50-100 \mathrm{mb}$ in a narrow $16-18 \mathrm{MeV}$ energy region where the (n,2n') cross section is a factor of $\approx 30$ larger. Estimated uncertainties in the ( $\left.n, 3 n^{\prime}\right)$ cross sections are given in Table 5. The corresponding neutron-emission spectra were obtained from the combined calculations, as outlined above in the context of the continuum inelastic - scattering processes. The present evaluated $\left(n, 3 n^{\prime}\right)$ cross sections are somewhat smaller than those of ENDF/B-V, 4 but well within the respective uncertainties. The differences will generally be of little applied concern, as they are all above $14 \mathrm{MeV}$.

Table 5. (n,3n') Cross-Section Uncertainties.

\begin{tabular}{cc}
\hline $\mathrm{E}_{\mathrm{n}}(\mathrm{MeV})$ & Uncertainty $(\%)$ \\
\hline 16.0 & 30 \\
18.0 & 15 \\
20.0 & 10
\end{tabular}

The $\left(n, 2 n^{\prime}\right)$ evaluation is largely based upon only two data sets which are reasonably consistent, but if significant improvements are desired, some careful new measurements are required. The $\left(n, 3 n^{\prime}\right)$ evaluation is essentially based upon a single data set and a statistical calculation, and new measurements are even more necessary for improvement here. 


\section{EVALUATED CHARGED-PARTICLE-EMITTING REACTIONS}

A number of charged-particle-emitting reactions are energetically possible, as outlined in Table 6.95 All of these reactions are greatly inhibited by the coulomb barrier and, as a consequence, the corresponding cross sections are very small. They will have a negligible effect in the vast majority of neutronic applications of the file, and so are included in the present evaluation largely as a matter of completeness. The relevant experimental information is sparse to non-existent, and thus the evaluation relies heavily upon the results of model calculations using the code CADE.88 The cross section uncertainties may be very large, easily a factor of two or more in many cases; therefore, they are not given numerically in the file.

Table 6. Q-values for charged-particle-emitting reactions.

Reaction

$$
\text { Q-value (MeV) }
$$

$\begin{array}{lr}(\mathrm{n}, \mathrm{p}) & +0.138 \\ \left(\mathrm{n} ; \mathrm{n}^{\prime}, \mathrm{p}\right) & -3.798 \\ \mathrm{n}, \mathrm{d}) & -1.573 \\ \left.\mathrm{n} ; \mathrm{n}^{\prime}, \mathrm{d}\right) & -8.941 \\ \mathrm{n}, \mathrm{t}) & -2.685 \\ \left.\mathrm{n} ; \mathrm{n}^{\prime}, \mathrm{t}\right) & -9.424 \\ \left.\mathrm{n},{ }^{3} \mathrm{He}\right) & -4.087 \\ \left.\mathrm{n} ; \mathrm{n}^{\prime},{ }^{3} \mathrm{He}\right) & -10.931 \\ \mathrm{n}, \alpha) & +9.648 \\ \left.\mathrm{n} ; \mathrm{n}^{\prime}, \alpha\right) & +3.144\end{array}$

A. (n,p) Reaction.

There apparently have been only four measurements of this cross section reported in the literature, $96-99$ all grouped near $14 \mathrm{MeV}$. All but one of the results are less than $1 \mathrm{mb}$. The three values that are relatively consistent give cross sections in the range $0.5-0.8 \mathrm{mb}$. The $14.0 \mathrm{MeV}$ result obtained with the CADE calculation is $\approx 0.22 \mathrm{mb}$. The calculated results were normalized to the average of the experimental values in the $\approx 14.0 \mathrm{MeV}$ region and used for the evaluation over the full energy range. The calculations did not include precompound contributions. Estimates of those contributions, using ALICE, ${ }^{99}$ suggest that they are factors of 4-5 larger than the sparse experimental values near $14.0 \mathrm{MeV}$. The weak experimental database and the calculational ambiguities indicate that the uncertainties of the evaluation are large (at least 100\%), but this should be of little note in the vast majority of neutronic applications. For example, the fission-spectrum-integral result, measured by Roy and Hawton, ${ }^{100}$ is only $0.005 \mathrm{mb}$; thus the reaction contributes 
very little to hydrogen production in fission-reactor systems. The cross sections of the present evaluation are approximately a factor of two smaller than those of ENDF/B-V 4 near $14.0 \mathrm{MeV}$. The present evaluated cross sections peak at $\approx 14.0 \mathrm{MeV}$ and then decrease with increasing energy, in contrast to those of ENDF/B-V which steadily increase with energy. The energy-dependent shape of the present evaluation is probably more realistic due to the physical nature of the calculations employed, although the directreactions, not considered in the calculations, will tend to increase the higher-energy cross sections.

\section{B. $\left(n ; n^{\prime} p\right)$ Reaction.}

No experimental information is available on this reaction; thus, the evaluation is based entirely upon the CADE 68 calculations, renormalized by the same factor used in the above $(\mathrm{n}, \mathrm{p})$ evaluation. The cross sections are small, less than $1 \mathrm{mb}$ below $\approx 15 \mathrm{MeV}$, and increase to $\approx 16 \mathrm{mb}$ at $20 \mathrm{MeV}$. The uncertainties associated with these values are very large, probably over $100 \%$. However, the effect on neutronic calculations will be negligibly small in most applications. In view of the small cross-section magnitudes, the associated neutron-emission spectra are described by simple isotropic temperature distributions. The ENDF/B-V4 file has no comparable entry.

C. (n,d) Reaction.

There is apparently no experimental evidence for this reaction below $20 \mathrm{MeV}$, and it is very difficult to measure in the presence of the $\left(n ; n^{\prime}, p\right)$ process. Therefore, the evaluation relies upon the CADE ${ }^{08}$ calculations. The cross section rises steadily to several $\mathrm{mb}$ at $20 \mathrm{MeV}$. This behavior is consistent with experimental evidence at $\approx 22 \mathrm{MeV},{ }^{101}$ which suggests that the sum of $(n, d)$ and $\left(n ; n^{\prime}, p\right)$ cross sections is several $m b$ at that energy. There is no comparable entry given in ENDF/B-V.4

D. $\quad\left(\mathbf{n} ; \mathbf{n}^{\prime}, \mathrm{d}\right)$ Reaction.

There is no experimental information; therefore, the cress sections are taken from CADE ${ }^{68}$ calculations, and the emission spectrum is described by a simple isotropic temperature distribution. The cross sections are less than $1 \mathrm{mb}$ below $20 \mathrm{MeV}$. There is no comparable ENDF/B-V4 file.

E. $(n, t)$ Reaction.

There is experimental evidence for a total tritium-production cross section at $\approx 22 \mathrm{MeV}$ of less than a few mb. ${ }^{101}$ This is consistent with the results of the CADE68 calculations used in the present evaluation which give a tritium-production cross section at $20 \mathrm{MeV}$ of $\approx 0.5 \mathrm{mb}$. There is no comparable ENDF/B-V 4 file.

F. $\quad\left(n ; n^{\prime}, t\right)$ Reaction.

There is no experimental information other than the total tritium-production result cited above. The evaluation relies entirely on $\mathrm{CADE}^{68}$ calculations for the cross sections, and the emitted-neutron spectra are described by simple isotropic temperature distributions. 
G. $\quad\left(n,{ }^{3} \mathrm{He}\right)$ Reaction.

Again, there is no experimental information, and the evaluation relies entirely upon CADE 88 calculations. The cross sections are very small (in the $\mu \mathrm{b}$ range). It is reasonable to expect the $\left(n ; n^{\prime},{ }^{3} \mathrm{He}\right)$ cross sections to be even smaller; thus they were ignored.

H. $(\mathrm{n}, \alpha)$ Reaction.

There are some experimental results near $14.0 \mathrm{MeV}^{98}, 99{ }^{\prime} 100$ '102, with cross-sections varying from $\approx 0.5$ to $1.2 \mathrm{mb}$. These are relatively consistent with the results of the CADE'8 calculations. Thus, the calculated results were slightly renormalized to improve the agreement with the average of the experimental values to obtain the evaluated cross sections. This approach ignores the possible presence of significant pre-compound contributions. The latter are probably not important below $15.0 \mathrm{MeV}$, as the statistical CADE calculations are reasonably representative of the measured values. The present evaluation is similar to that of ENDF $/ \mathrm{B}-\mathrm{V}^{4}$ at $14.0 \mathrm{MeV}$, and smaller by approximately a factor of two at $20 \mathrm{MeV}$. The differences are probably within the respective large evaluation uncertainties (e.g., $100 \%$ or more at many energies).

I. $\left(\mathbf{n} ; \mathbf{n}^{\prime}, \alpha\right)$ Reaction.

The present evaluation relies entirely upon the CADE calculations, normalized by the same factor used in the above $(n, \alpha)$ evaluation, to obtain the evaluated cross sections. The neutron-emission spectra are described by simple isotropic temperature distributions. There may be a significant pre-compound contribution which was not taken into account. This is a matter of calculational speculation. The uncertainties associated with this portion of the evaluation are very large (probably over 100\%). The results are qualitatively similar to those given in ENDF/B-V.4

\section{EVALUATED PHOTON-PRODUCTION REACTIONS}

The photon-production data are made up of contributions from the $(\mathbf{n}, \gamma),\left(\mathbf{n} ; \mathbf{n}^{\prime}, \gamma\right)$ (to specific levels), and a continuum from all other photon-producing reactions.

Photon production for the $(n, \gamma)$ reaction is dealt with by providing energydependent photon multiplicities and spectra. The spectrum of neutrons from the neutroncapture reaction was taken from the work of Orphan et al. ${ }^{103}$ at thermal-neutron energy. The average energy of the spectrum was determined and divided into the $Q$-value for the reaction in order to determine the low-energy photon multiplicity. The same spectrum was used at $20 \mathrm{MeV}$, with the multiplicity adjusted to conserve energy.

For photons associated with inelastic scattering to specific levels, Warren's code CASCADE, 104 which incorporates the method used in Reffo's BRANCH code, ${ }^{105}$ was used to obtain the energy-dependent cross sections for specific photons resulting from deexcitation of the discrete levels excited by inelastic neutron scattering.

For all other reactions, the photon production cross sections and spectra were calculated using the R-parameter formalism of Perkins et al.106 The R-parameter formalism requires formal representation of energy distributions for all secondary particles 
(i.e., charged-particles as well as neutrons), in order to calculate the photon-production cross sections and spectra. Since the ENDF/B-VI formats and procedures allow for secondary charged-particle distributions is File 5 only if there is a single secondary particle, the file was translated into the ENDL format where energy distributions for all secondary particles can be represented. The $R(U)$ values used were taken from the "best curve" of Fig. 3 of Ref.106.

After entering the calculated photon-production data, energy conservation was calculated and verified to within $10 \%$ for all incident neutron energies.

\section{SUMMARY COMMENTS}

The energy-averaged neutron total cross sections are quite well known (to $\approx 1-2 \%$ ) from $\approx 0.1-15 \mathrm{MeV}$. Above $\approx 15 \mathrm{MeV}$ additional careful measurements would add confidence to this evaluation. The resonance parameterization is believed to be reasonably descriptive of observations, but most of the latter were not made to contemporary standards. Thus, new resonance measurements, and accompanying interpretations, would be useful. Energy -averaged elastic scattering is very well known to $\approx 10 \mathrm{MeV}$. However, there is need for careful differential-elastic-scattering measurements at higher energies, particularly as this is a region of changing optical potentials, and as the nonelastic cross section is a major consideration in the higher-energy aspects of the evaluation. Discrete-inelastic-scattering data are marginal. New measurements are needed for significant evaluation improvements, but they will not be trivial due to the high density of levels at excitations of several $\mathrm{MeV}$. Energy-average radiative-capture data are sparse and discrepant. As a consequence, the capture evaluation is little more than a qualitative model construction. An improved capture evaluation will require new measurements that will not be easy to do. Fortunately, for most of the neutronic applications, the capture cross sections are small. The $\left(n, 2 n^{\prime}\right)$ cross sections are very large and reasonably well defined from threshold to $20 \mathrm{MeV}$ by two comprehensive data sets which are quite consistent in the energy region of overlap. The estimated cross-section uncertainties are as small as $\approx 5 \%$. It will be difficult to improve upon the $\left(n, 2 n^{\prime}\right)$ evaluation without extensive and precise new measurements which must be carefully justified, in view of the relatively good status of the $\left(n, 2 n^{\prime}\right)$ cross sections. A number of charged-particleemitting channels are open, and the respective cross sections and emitted-neutron spectra are very pcorly known. However, all of these cross sections are very small, to at least $15.0 \mathrm{MeV}$, and thus not a concern in the vast majority of applications.

The present evaluation is believed to be an improvement on the prior ENDF/B-V4 evaluation, but that improvement is a matter of refinement, with no large discrepancies in aspects of the evaluation important to neutronic applications. That is not surprising as, excepting neutron scattering, the data bases underlying the two evaluations are quite similar. The models used in the present evaluation have been refined from past practice, particularly including a reasonable physical foundation. The present evaluation gives attention to both qualitative and quantitative uncertainty specifications, depending on the status of the relevant databases. These will allow an estimate of sensitivities in neutronic applications. 


\section{REFERENCES}

1. R. Kinsey, ed., National Nuclear Data Center Report, ENDF-102, (1979); and subsequent revisions.

2. S. F. Mughabghab, Neutron Cross Sections Vol. 1, Part B, Academic Press Inc. New York, (1984); also S. Mughabghab and C. Dunford, private communication (1982).

3. D. Cullen, Lawrence Livermore National Laboratory Report, UCRL-50400, Vol. 17, Part C (1979).

4. Evaluated Nuclear Data File B, Version-V (ENDF/B-V), National Nuclear Data Center, Brookhaven National Laboratory; also Argonne National Laboratory Report, ANL/NDM-51 (1980).

5. A. Smith et al., Nucl. Sci. and Eng. $4163(1970)$, set $1, \delta_{\text {sys }}=2 \%$.

6. A. Smith et al., Nucl. Sci. and Eng. $\underline{41} 63(1970)$, set $2, \delta_{\text {sys }}=2 \%$.

7. D. Foster and D. Glasgow, Phys Rev. C3 $576(1971), \delta_{\text {sys }}=1.5 \%$.

8. J. Harvey, private communication, data at NNDC (1985), $\delta_{\text {sys }}=1.5 \%$.

9. M. Divadeenam et al., Thesis, Duke University (1968), $\delta_{\text {sys }}=3.0 \%$.

10. U. Singh et al., Phys. Rev. $\underline{\text { C13 }} 124$ (1976), abandoned, too low energy.

11. P. Guenther et al., Nucl. Sci. and Eng. $\underline{75} 69(1980)$, set $1, \delta_{\text {sys }}=1.5 \%$.

12. Set 2 from Ref. $11, \delta_{\text {sys }}=1.5 \%$.

13. J. Coon et al., Phys. Rev. $\underline{88} 562(1952), \delta_{\text {sys }}=1.0 \%$.

14. L. Goodman, Phys. Rev. $\underline{88} 686(1952), \delta_{\text {sys }}=2.5 \%$.

15. N. Nereson and S. Darden, Phys. Rev. $\underline{89} 775$ (1953), abandoned, large uncertainties.

6. R. Day and R. Henkel, Phys. Rev. $\underline{92} 358(1953), \delta_{\text {sys }}=2 . \%$.

17. J. Peterson et al., Phys. Rev. $\underline{120} 521(1960), \delta_{\text {sys }}=1.8 \%$.

18. A. Bratenahl et al., Phys. Rev. 110927 (1958), $\delta_{\text {sys }}=2.0 \%$.

19. M. Walt and J. Beyster, Phys. Rev. $98677(1955), \delta_{\text {sys }}=2.5 \%$.

20. C. Hibdon and A. Langsdorf, Phys. Rev. $\underline{98} 223$ (1955), abandoned, toc low energy. 
21. R. Fields et al., Phys. Rev. 71508 (1947), discrepant, abandoned.

22. A. Carlson and H. Barschall, Phys. Rev. 1581142 (1965), $\delta_{\text {sys }}=1.5 \%$.

23. J. Garg et al., EANDC(US)-54 (1964), abandoned, too low energy.

24. D. Miller et al., Phys. Rev. $\underline{88} 83$ (1952), discrepant results, abandoned.

25. J. Gibbons, Phys. Rev. 1021574 (1956), too low energy, abandoned.

26. S, Snowdown and W. Whitehead, Phys. Rev. 941267 (1954), discrepant, abandoned.

27. R. Day, Data from NNDC, priv. com., discrepant, abandoned.

28. H. Barschall et al., Phys. Rev. $\underline{73} 659$ (1948), $\delta_{\text {sys }}=2.5 \%$.

29. H. Barschall et al, Phys. Rev. $\underline{76} 1146(1949), \delta_{\text {sys }}=2.5 \%$.

30. C. Kimball et al., Report, WASH-745 7 (1949), discrepant, abandoned.

31. F. Seidel et al., Report, WASH-1074 $36(1967), \delta_{\text {sys }}=2.5 \%$.

32. S. Cierjacks et al., Kernforschungszentrum-Karlsruhe Report, KFK-1000 $(1968), \delta_{\text {sys }}=1.5 \%$.

33. G. Deconninck et al., Bull. Acad. Roy. Belgium 44451 (1958), discrepant, abandoned.

34. F. Manero, Nucl. Phys. $\underline{65} 419$ (1965), discrepant, abandoned.

35. P. Kuijper et al., Nucl. Phys. A181 545 (1972), $\delta_{\text {sys }}=2.0 \%$.

36. L. Drigo et al., Nuovo Cimento A 13867 (1973), $\delta_{\text {sys }}=2.5 \%$.

37. V. Giordano et al., Nucl. Phys. $\underline{A 302} 83(1978), \delta_{\text {sys }}=2.2 \%$.

38. P. Engelstaff, priv. com. to NNDC (1955), $\delta_{\text {sys }}=3.0 \%$.

39. M. Ageno et al., Nuovo Cimento $\underline{10} 281$ (1953), $\delta_{\text {sys }}=3.0 \%$.

40. M. Mazari et al., Geneva Conf. (1958), discrepant, abandoned.

41. I. Angeli et al., Acta. Phys. Acad. Sci. Hungary $\underline{30} 115(1971), \delta_{\text {sys }}=2.5 \%$.

42. C. Ai and J. Chou, Nucl. Sci. Formosa 11179 (1974), abandoned, discrepant.

43. R. Maggi et al., INIS-MF-1743 (1975), discrepant, abandoned.

44. A. Tutubalin et al., Kiev Conf. (1977), $\delta_{\text {sys }}=2.1 \%$. 
45. M. Khaletskij, Doklady Akad. Nauk. USSR $\underline{13} 305$ (1956), $\delta_{\text {sys }}=3.0 \%$.

46. Ju. Dukarevich et al., Nucl. Phys. $\underline{A 92} 433$ (1967), $\delta_{\text {sys }}=2.5 \%$.

47. G. Gorlov et al., Yadernaya Fizika $\underline{6} 910$ (1967).

48. W. P. Poenitz, Brookhaven National Laboratory Report, BNL-NCS-51363 (1981); as modified by M. Sugimoto (1987).

49. A. Smith, P. Guenther and R. Lawson, Argonne National Laboratory Report, ANL/NDM-100 (1987).

50. A. Smith and R. Lawson, to be published.

51. R. D. Lawson, P. T. Guenther and A. B. Smith, Phys. Rev. C36 1298 (1987).

52. G. R. Satchler, Direct Nuclear Reactions, Clarendon, Oxford (1983).

53. C. Lederer and V. Shirley, eds., Table of Isotopes, $7^{\text {th }}$ Edition, John Wiley and Sons Inc. New York (1978).

54. G. C. Wick, Atti R. Accad. Ital. Memorie $\underline{13} 1203$ (1943).

55. S. Chiba and A. Smith, to be published (1989).

56. Ju. Degtjarev and V. Protopopov, IZV. Akad. Nauk SSSR, Ser. Fiz, $\underline{35} 2341$ $(1971),\left(n ; n^{\prime}, \gamma\right)$.

57. D. Boder et al., IZV. Akad. Nauk SSSR, 31327 (1967), (n;n' $\gamma)$.

58. Ju. Degtjarev and V. Protopopov, Atom. Energiya $\underline{23} 568(1967),\left(n ; n^{\circ}, \gamma\right)$.

59. W. Seeliger, Kern. Energy, $20174(1977),\left(n, n^{\prime}\right)$.

60. E. Eliot et.al., Phys. Rev. $\underline{94} 144(1954),\left(n ; n^{\prime}, \gamma\right)$.

61. E. Almen-Ramstrom, Aktiebolaget Atomenergi Report, AE-503 (1975), $\left(\mathbf{n}, \mathbf{n}^{\prime}\right)$.

62. R. Kiehn and C. Goodman, Phys. Rev. $\underline{95} 989(1954),\left(n ; n^{\prime}, \gamma\right)$.

63. A. Smith et al., Nucl. Sci. and Eng. $4163(1970),\left(n, n^{\prime}\right)$.

64. P. Guenther et al., Nucl. Sci. and Eng. $\underline{75} 69$ (1980): (n, $\left.n^{\prime}\right)$.

65. A. Gilbert and A. Cameron, Can. J. Phys. $\underline{43} 1446$ (1965).

66. A. Smith, D. Smith, P. Guenther, J. Meadows, R. Lawson, R. Howerton, T. Djemil and B. Micklich, Argonne National Laboratory Report, ANL/NDM-105 (1988). 
67. P. T. Guenther, to be published.

68. D. Wilmore, Harwell Report, AERE-R-11515 (1984).

69. M. Blann, Livermore National Laboratory Report, UCID-20169 (1984).

70. P. T. Guenther et al., to be published.

71. J. Perkin et al., Proc. Phys. Soc. $\underline{72} 505$ (1958).

72. J. Voignier et al., Nucl. Sci. and Eng. $\underline{93} 43$ (1986).

73. M. Diksic et al., Acta. Phys. Acad. Sci. Hungary, 28257 (1970).

74. J. Csikai et al., Nucl. Phys. $\underline{\mathbf{A 9 5}} 229$ (1967).

75. M. Budnar et al., Nucl. Instr. and Methods $\underline{44} 292$ (1966).

76. R. Macklin and J. Halpern, Phys. Rev. C14 1389 (1976), broad energy bins.

77. W. Gibbons, Phys. Rev. 122182 (1961).

78. R. Booth et al., Phys. Rev. 112226 (1958).

79. L. Seren et al., Phys. Rev. $\underline{72} 888$ (1947).

80. A. Poularikas and R. Fink, Phys. Rev. 115989 (1959), upper limit only.

81. B. Diven et al., Phys. Rev. 120556 (1960).

82. C. Kimball et al., Report, WASH-745 7 (1957).

83. I. Bergquist et al., Nucl. Phys. $\underline{\text { A120 }} 161$ (1968).

84. P. Moldauer, computer code ABAREX, private communication (1982).

85. L. Veeser et al., Proc. Inter. Conf. on the Interaction of Neutrons with Nuclei 2 1351 (1976).

86. V. Ashby et al., Phys. Rev. 111616 (1958).

87. L. Rosen et al., Phys. Rev. 107824 (1957).

88. G. Butler et al., Los Alamos Report, LA-9381 (1982).

89. J. Frehaut et al., Proc. Kiev Conf. (1975).

90. E. Feicht and H. Vonach, Nukleonics 1058 (1967).

91. A. Adam et al., Nucl. Phys. $\underline{49} 489$ (1963). 
92. F. Deak et al. Acta. Phys. Acad. Sci. Hung. $\underline{38} 209$ (1975).

93. N. Flerov and K. Talyzin, Atom. Energy $\underline{5} 657$ (1958).

94. P. Bornemisza-Pauspertl et al., Radiochem. and Radioanaly. Let. $\underline{32} 277$ (1978).

95. Lawrence Livermore National Laboratory Table of Q-values, available from one of the authors (RJH).

96. R. Coleman, B. Hawker, L. O'Conner and J. Perkin, Proc. Phys. Soc. (London) 73215 (1959).

97. S. Mukherjee et al., Proc. Sym. Nucl. Phys., Waltair, 289 (1969).

98. A. Poularikas and R. Fink, Phys. Rev. 115989 (1959).

99. S. Mukherjee, A. Ganful and N. Mejunder, Proc. Phys. Soc. (London) 77508 (1961).

100. J. Roy and J. Hawton, Atomic Energy of Canada Report, CRC-1003 (1969).

101. S. Qaim, R. Wolfe and G. Stocklin, Nucl. Chem. $\underline{36} 3639$ (1974), also Nucl. Phys. A295 150 (1978).

102. E. Paul and R. Clark, Can. J. Phys. 31267 (1953).

103. V. J. Orphan, N. C. Rasmussen and T. L. Harper, Line and continuum $\gamma$-ray yields from thermal-neutron capture in 75 elements, Gulf General Atomic Report, GA-10248/DASA 2570 (1970).

104. W. E. Warren, R. J. Howerton and G. Reffo, CASCADE Cray program for $\gamma$-production from discrete level inelastic scattering, Lawrence Livermore Nuclear Data Group Internal Report, PD-134 (1986), unpublished.

105. G. Reffo, IDA - A modular system of nuclear model codes for the calculation of cross sections for nuclear reactors, Centro Ricerche Energia, Bologna (1980), private communication.

106. S. T. Perkins, R. C. Haight and R. J. Howerton, Nucl. Sci. and Eng. $\underline{57} 1$ (1975). 


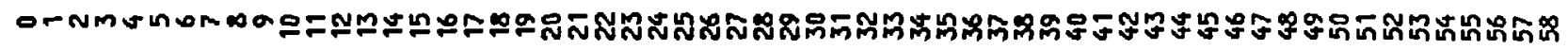

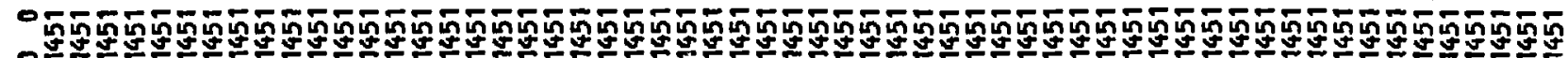

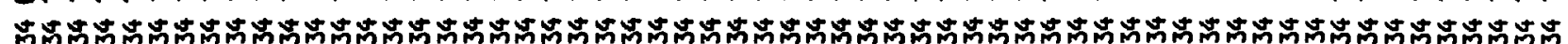

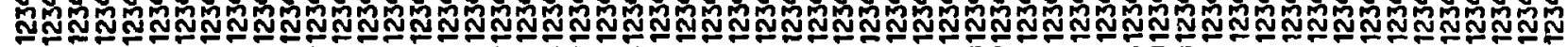

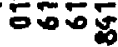
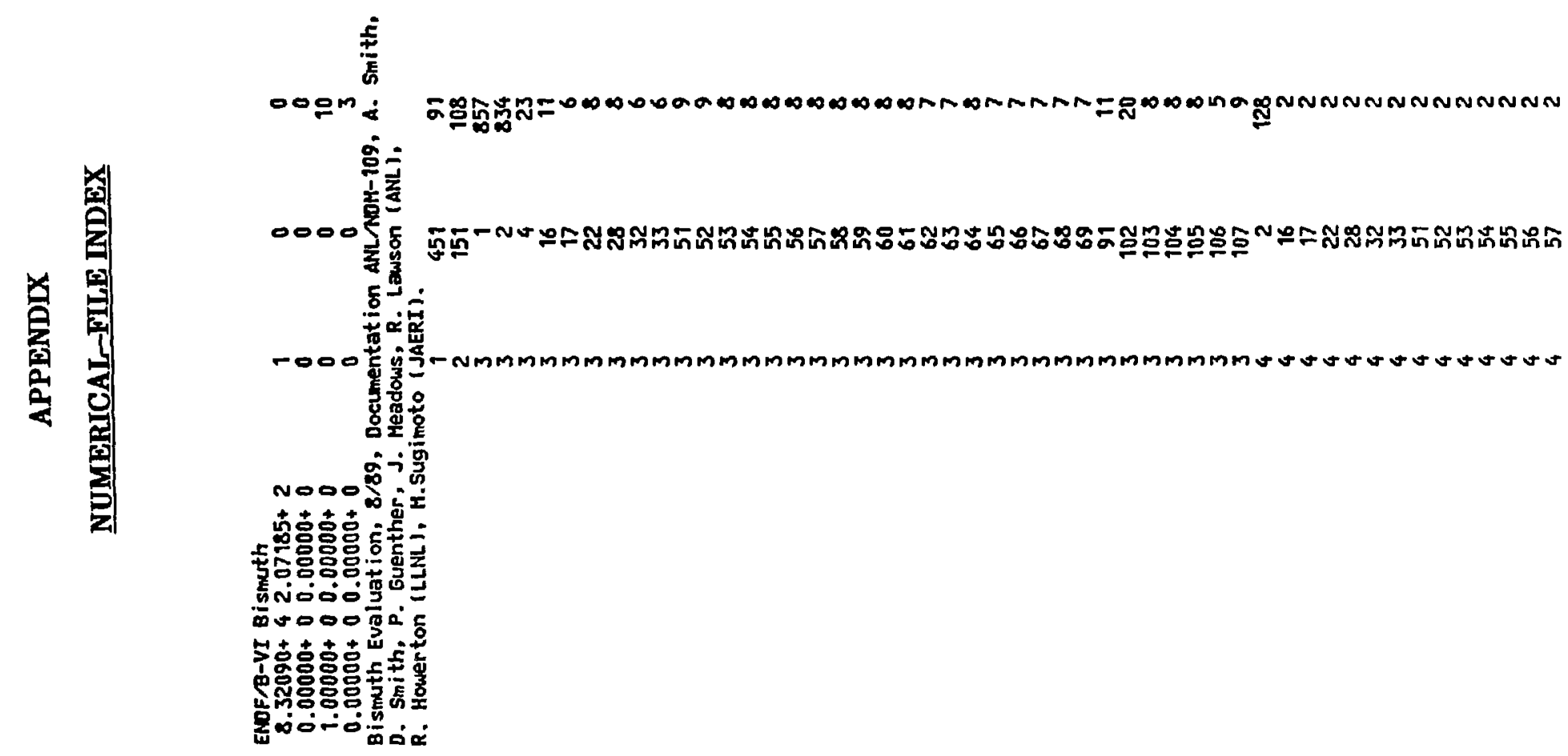

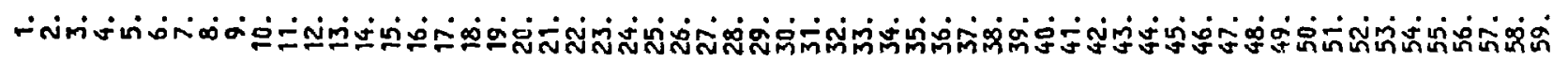


60.
61.
62.
63.
64.
65.
66.
67.
68.
69.
70.
71.
72.
73.
74.
75.
76.
77.
78.
79.
80.
81.
82.
83.
84.
85.
86.
87.
88.
89.
90.
91.
92.
93.

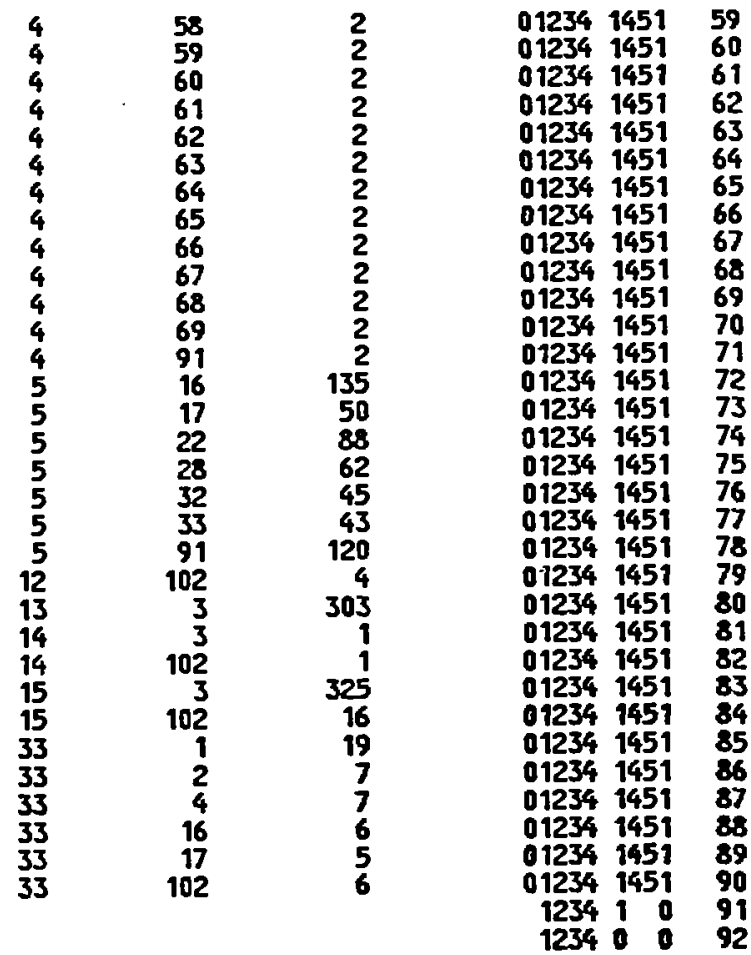

